\title{
The vascular plants of Chujado Island (Jeju-do), Korea
}

\section{Eun-Mi SUN, Yu Chul PARK', Kang-Hyup LEE, Kuk Hwa SONG, Dong Chang SON*}

Division of Forest Biodiversity and Herbarium, Korea National Arboretum, Pocheon 11186, Korea ${ }^{1}$ Team of National Ecosystem Survey, National Institute of Ecology, Seocheon 33657, Korea (Received 10 October 2019; Revised 18 November 2019; Accepted 14 December 2019)

\begin{abstract}
To identify the flora of the vascular plant on Chujado Island in Chuja-myeon, Jeju-si, Jeju Province, Korea, eleven field surveys were conducted between April to September of 2013 and from May to September of 2018. The results of the surveys identified 558 taxa, with 97 families, 311 genera, 481 species, two subspecies, 65 varieties, and ten forms of vascular plants on Chujado Island. Of the 558 taxa identified, seven were endemic species and eight were rare plants according to the Korea Forest Service. Furthermore, 109 of the taxa identified were from floristic environments, while 62 were considered to be naturalized plants, with three of these considered to be invasive and causes of ecosystem disturbances. The highest degree of similarity among the plants from seven sites adjacent to Chujado Island was found on Saengil Island, at 51.7\%.
\end{abstract}

Keywords: Chujado Island, flora, plant geography, Sørensen’s Coefficients for community similarity

추자도는 한반도 남부 지역과 제주도 해상 중간 지점에 위치하여, 난류성 어류의 회유지, 철새들의 번식지, 어선 들의 중간 보급지로 이용되는 지역이다. 식물지리학적인 측면에서도 추자도는 제주도와 서남해안지방의 연륙교 에 해당되는 지역으로 매우 중요하며, 추자도의 식물상 역시 양 지역의 식물상과 밀접한 관계가 있을 것으로 추 정된다.

추자도의 식물연구는 1940년 『전라남도 식물』에 분포 지가 ‘추자도'로 명시된 것을 시초로 하여(Yang, 2006), Lee (1969)에 의해서 63과 134속 167종이 보고되면서 본격 적인 식물상 연구가 시작되었다. 이후 Lee and Oh (1969)는 7과 49속 66종, Park (1969)은 7과 11속 12종, Lim et al. (1982)은 86과 210속 228종 38변종 4품종을 각각 보고하였 으며, 이후에도 Jung et al. (1986)에 의해 389분류군, Yang (2006)에 의해 97과 289속 384종 46변종 4변종 2품종 등 꾸 준한 식물상 연구가 지속되어 왔다. 최근 연구로는 Ko et al. (2013)이 264분류군, Hong et al. (2014)이 90과 272속 386 종 2아종 54변종 7품의 449분류군을 조사한 바 있다. 그러 나 그 동안의 식물상 연구는 식물목록 작성을 중심으로 연구가 수행되었으며, 특기할만한 식물에 대한 논의가 없 을 뿐만 아니라, 확인된 식물에 대한 증거표본이 제시되 어 있지 않고, 오동정, 분류학적 이명 등 실체가 불확실한 분류군들이 다수 포함되어 있어 추자도의 정확한 관속식
물상을 파악하는 데 어려움이 있다. 본 연구는 추자도 일 대를 조사하여 신뢰도 있는 관속식물상을 제시하고, 식물 종의 효율적인 관리와 식물다양성 종 보존 전략 수립을 위한 기초자료를 제공하고자 수행되었다.

\section{재료 및 방법}

\section{조사지 개황}

추자도는 지리적으로 북위 $33^{\circ} 55^{\prime} 32^{\prime \prime}-33^{\circ} 22^{\prime} 36^{\prime \prime}$, 동경 $126^{\circ} 22^{\prime} 25^{\prime \prime}-126^{\circ} 23^{\prime} 05^{\prime \prime}$ 사이에 위치하고, 행정구역상으로 는 제주특별자치도 제주시 추자면에 속한다. 추자도는 상 추자도와 하추자도 2 개의 섬으로 이루어져 있고, 총 면적 은 $7.03 \mathrm{~km}^{2}$ 에 이른다. 인근에는 42개의 유인도(4개)와 무 인도(38개)가 추자군도를 형성한다. 본 섬은 자연경관이 수려한 곳이 많고, 섬 주변에는 다양한 어종들이 서식하 여 관광객 및 바다낚시를 하기위한 사람들로 유입이 매우 많은 섬이다. 상추자도는 대서리지역에 추자항이 위치하 고, 항구 주변에 마을이 형성되어 인구 밀도가 가장 높다. 상추자도 남쪽에는 큰산 $(142 \mathrm{~m})$ 과 북쪽에는 봉굴레산 $(85.5 \mathrm{~m})$ 이 각각 솟아 있고, 남서쪽 해안은 오랜 세월 파도 에 의해 암석이 노출이 심한 나바론절벽이 있다. 하추자도 는 돈대산 $(163.9 \mathrm{~m})$ 을 중심으로 동쪽에 추석산 $(155.7 \mathrm{~m})$, 남쪽에 대왕산 $(124.9 \mathrm{~m})$ 과 석두청산 $(86 \mathrm{~m})$ 이 각각 위치하

\footnotetext{
*Author for correspondence: sdclym@korea.kr
} 


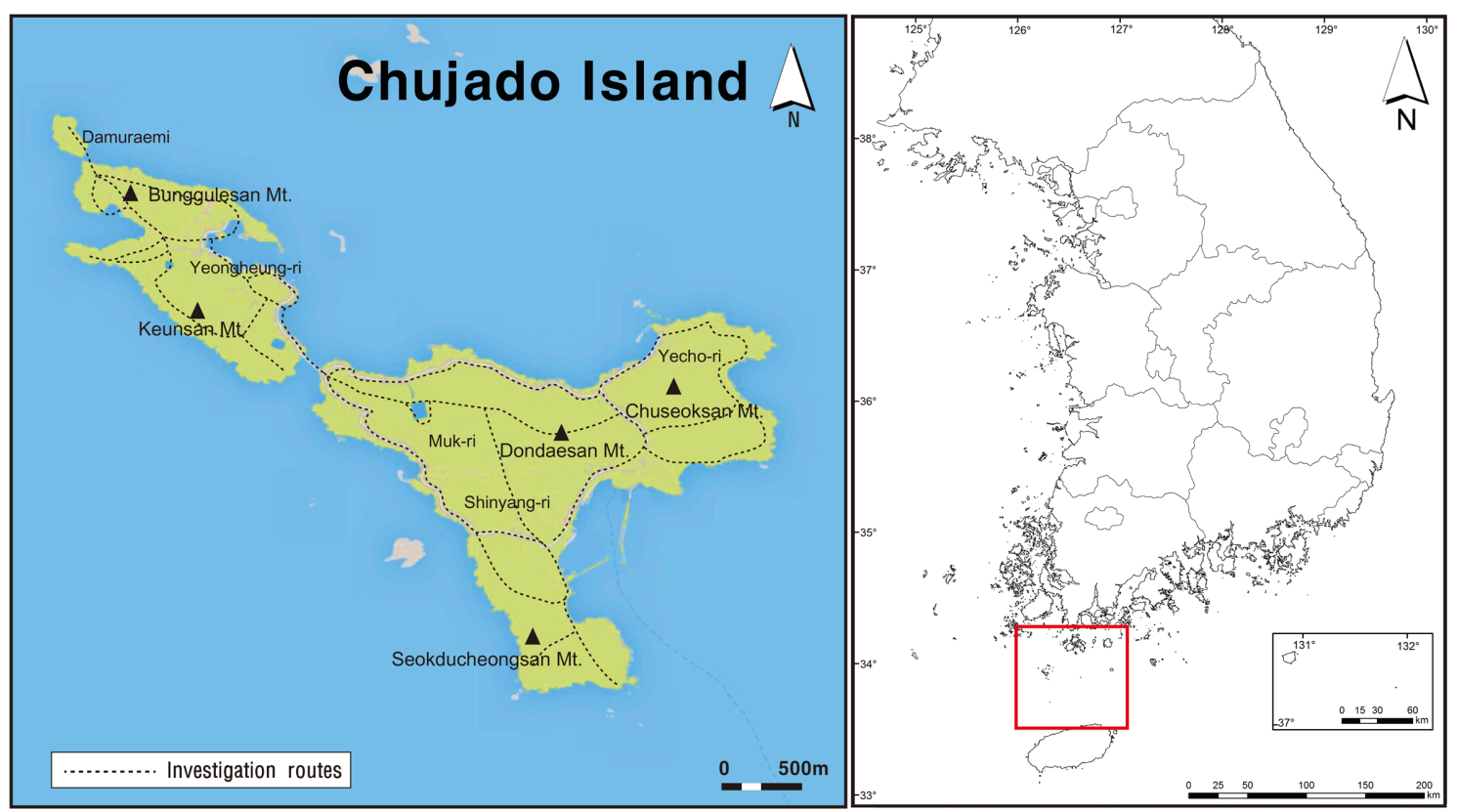

Fig. 1. Investigated area of Chujado Island.

고 있으며, 크고 높은 산이 없어 대부분 낮은 산지를 이루 고 있으나 산의 경사는 가파른 편이다. 식물구계학적으로 는 일화식물구계(Sino-Japanese Region), 온대아구계의 한 국구에 속한다(Lee and Yim, 1978). 기후는 온난다습한 해 양성 기후이고, 가장 인접한 완도 기상관측소를 기준으로 2018 년 연평균기온은 $14.8^{\circ} \mathrm{C}$ (최저 $11.1^{\circ} \mathrm{C}$, 최고 $19.2^{\circ} \mathrm{C}$ )이 며, 연평균강수량은 $1431.4 \mathrm{~mm}$ 이고, 평균습도는 $74.1 \%$ 이 다(Korea Meteotological Administration, 2019).

\section{조사방법}

조사범위는 행정구역상 제주특별차지도 제주시 추자 면에 위치한 추자도 일대로 현지조사는 2013년 4월부터 동년 9월, 2018년 5월부터 동년 9월까지 총 11일에 걸쳐 식 물조사를 수행하였다(Fig. 1, Table 1). 또한 국립수목원 산 림생물표본관(KH)에 석엽표본으로 소장되어 있는 추자 도 일대의 표본을 재동정하여 목록에 추가 작성하였다. 특히 하추자도를 중심으로 산의 능선지역과 계곡부, 해변 지역을 집중적으로 조사하였다. 증거표본 확보를 위하여 기본적으로 동일종에 대해 1 개체 이상을 수집하여 확증 표본(voucher specimen)으로 제작하여 $\mathrm{KH}$ 에 수장하거나 photography sample로 제작하였다. 학명과 국명은 국가표 준식물목록 개정판(Korea National Arboretum, 2017)을 기 준으로 배열하고 표기하였다. 주요 식물들은 한국특산식 물목록(Chung et al., 2017), 희귀식물목록(Korea National Arboretum, 2008), 환경부 식물구계학적 특정식물목록 (Ministry of Environment 2014), 귀화식물목록(Park, 1995, 2009) 등에 따라 식물목록을 작성하였다. 추자도와 주변
도서에 출현하는 식물종들의 유사도 지수(Similarity index)를 비교·분석하기 위해 인근 도서의 식물상 논문의 식물목록을 참고하였으며(Kim et al., 2005; Lee, 2005; Kang, 2011; Oh and Beon, 2011; Son et al., 2013; Park, 2019), 식물상 유사도는 Sörensen index (Sörensen, 1948)의 유사계 수(Sørensen's Coefficients for community similarity, CCs) 수 식을 기초로 하여 분석하였다.

$$
\mathrm{CCs}=\frac{2 \mathrm{C}}{\mathrm{S}_{1}+\mathrm{S}_{2}}
$$

$\mathrm{CCs}=$ Sørensen's Coefficients for community similarity

$\mathrm{S}_{1}=$ Numbers of species in the community 1

$\mathrm{S}_{2}=$ Numbers of species in the community 2

$\mathrm{C}=$ Numbers of species in the both communities

\section{결과 및 고찰}

\section{관속식물상}

추자도에는 관속식물은 총 97과 311속 481종 2아종 65 변종 10 품종의 558 분류군이 분포하는 것으로 조사되 었으며(Table 2), 이는 한반도 관속식물 4,071종(Lee, 1996)의 $13.7 \%$ 에 해당된다. 분류군별로는 양치식물이 34 분류군(6.1\%), 나자식물이 4 분류군 $(0.7 \%)$, 피자식물 은 520 분류군으로 이 중 쌍자엽식물은 387 분류군 (69.4\%), 단자엽식물은 133 분류군(23.8\%)으로 나타났 다(Appendix 1).

멸종위기식물 II등급인 석곡(Dendrobium moniliforme 
Table 1. Investigation dates and routes of the floristic study of Chujado Island.

\begin{tabular}{|c|c|c|}
\hline No. & Date & Investigation routes \\
\hline 1 & 24 Apr 2013 & $\begin{array}{l}\text { A: Muk-ri } \rightarrow \text { Chuja-gyo (bridge) } \rightarrow \text { Seokducheongsan Mt. } \\
\text { B: Yecho-ri } \rightarrow \text { Dondaesan Mt. } \rightarrow \text { Chuja-gyo (bridge) } \\
\text { C: Jeongda lounge } \rightarrow \text { Lighthouse Observatory } \rightarrow \text { Cemtery } \rightarrow \text { Coastal }\end{array}$ \\
\hline 2 & 25 Apr 2013 & $\begin{array}{l}\text { A: Daeseo-ri } \rightarrow \text { Yongdeung-bong } \rightarrow \text { Observatory } \\
\text { B: Daeseo-ri } \rightarrow \text { Bonggollesan Mt. } \rightarrow \text { Hupo }\end{array}$ \\
\hline 3 & 1 Jul 2013 & $\begin{array}{l}\text { A: Muk-ri } \rightarrow \text { Dondaesan Mt. } \rightarrow \text { Chuja-gyo (bridge) } \rightarrow \text { Yecho-ri } \rightarrow \text { Coastal Road } \\
\text { B: Chuja-gyo (bridge) } \rightarrow \text { Dondaesan Mt. } \rightarrow \text { Yecho-ri } \\
\text { C: Yeongheung-ri } \rightarrow \text { wetland } \rightarrow \text { Sinyang-ri } \rightarrow \text { Seokjimeori }\end{array}$ \\
\hline 4 & 2 Jul 2013 & $\begin{array}{l}\text { A: Shinyang-ri } \rightarrow \text { Daewangsan Mt. } \\
\text { B: Chuja lighthouse } \rightarrow \text { Nabaron cliff } \rightarrow \text { Yongdeung-bong } \rightarrow \text { Bonggollesan Mt. } \\
\text { C: Chuja lighthouse } \rightarrow \text { Army unit } \rightarrow \text { Coastal road } \rightarrow \text { Damurami } \rightarrow \text { Cemtery }\end{array}$ \\
\hline 5 & 23 Sep 2013 & $\begin{array}{l}\text { A: Yeonngheung-ri } \rightarrow \text { Chuja-gyo (bridge) } \rightarrow \text { Lighthouse } \\
\text { B: Muk-ri } \rightarrow \text { Dondaesan Mt. } \rightarrow \text { Yecho-ri } \rightarrow \text { Yecho samgeori } \\
\text { C: Passenger terminal } \rightarrow \text { Forest road of Sinyang-ri } \rightarrow \text { Seokducheongsan Mt. }\end{array}$ \\
\hline 6 & 24 Sep 2013 & Yecho-ri $\rightarrow$ Shinyang-ri $\rightarrow$ Mojini seaside \\
\hline 7 & 29 May 2018 & Chuja-myeon office $\rightarrow$ Bunggullesan Mt. $\rightarrow$ Goindol $\rightarrow$ Sunhyo-gak \\
\hline 8 & 30 May 2018 & A: Yeonheung-ri $\rightarrow$ Nabaron-cliff $\rightarrow$ Keunsan Mt. $\rightarrow$ Chuja lighthouse $\rightarrow$ Myeon office \\
\hline 9 & 31 May 2018 & Hachujado Island $\rightarrow$ Fresh water facility \\
\hline 10 & 4 Sep 2018 & $\begin{array}{l}\text { A: Yecho-ri } \rightarrow \text { Dondaesan Mt. } \rightarrow \text { Muk-ri } \rightarrow \text { Sinyang-ri } \\
\text { B: Yecho-ri nursing hospital } \rightarrow \text { Chuseoksan Mt. } \rightarrow \text { Hoenggyeonghanui-grave } \rightarrow \text { Mojini seaside }\end{array}$ \\
\hline 11 & 5 Sep 2018 & Shinyang-ri seaside \\
\hline
\end{tabular}

Table 2. The summary of vascular plants in Chujado Island.

\begin{tabular}{ccccccccc}
\hline \hline Taxa & Fam. & Gen. & Sp. & Subsp. & Var. & For. & Total & Percentage \\
\hline Pteriodophyta & 8 & 18 & 32 & 0 & 2 & 0 & 34 & 6.1 \\
Gymnospermae & 2 & 4 & 3 & 0 & 1 & 0 & 4 & 0.7 \\
Angiospermae & 87 & 289 & 446 & 2 & 62 & 10 & 520 & - \\
Dicotyledonae & 76 & 217 & 338 & 2 & 40 & 7 & 387 & 69.4 \\
Monocotyledonae & 11 & 72 & 108 & 0 & 22 & 3 & 133 & 23.8 \\
Total & 97 & 311 & 481 & 2 & 65 & 10 & 558 & 100 \\
\hline
\end{tabular}

(L.) Sw.)과 솔잎란(Psilotum nudum (L.) P.Beauv.)은 남해안 과 제주도 일대에 제한적으로 생육하는 식물종으로 $\mathrm{Lim}$ et al. (1982)에 의해 상추자도와 하주차도에 분포가 보고 된 바 있다. 그러나 본 조사를 비롯하여 Yang (2006)과 Hong et al. (2014) 등을 포함한 최근 연구에서는 이들의 실 체가 확인되지 않았으며, 두 종 모두 식물분류학 및 식물 지리학적으로 학술적 가치가 매우 높은 중요한 식물이므 로 향후 보다 세밀한 조사가 요망된다.

추자도에는 섬오갈피나무(Eleutherococcus gracilistylus (W. W. Sm.) S. Y. Hu), 가는쇠고사리(Arachniodes aristata
(G. Forst.) Tindale), 제주산딸기(Rubus nishimuranus Koidz.), 해변노박덩굴(Celastrus orbiculatus Thunb. var. punctata (Thunb.) Rehder), 삼도하수오(Fallopia koreana B. U. Oh \& J. G. Kim)등과 같은 제주도아구 또는 남부아구에만 분포 하는 식물들이 추자도 일원에 생육하고 있어 식물지리학 적인 측면에서 학술적 중요성이 매우 큰 지역임을 단적으 로 보여준다. 이처럼 추자도는 제주도와 서남해안의 식물 상을 잇는 연륙교로서 학술적 중요도가 매우 높은 지역이 다. 앞으로 단편성이 아닌 지속적인 모니터링에 기반한 보완조사 및 기초자료 확보가 필요하다. 
Table 3. The list of endemic plants to Korea in Chujado Island designated by Korea Forest Service.

\begin{tabular}{clc}
\hline \hline Family name & Taxa & Korean name \\
\hline Ulmaceae & Celtis choseniana Nakai & 검팽나무 \\
& Rubus schizostylus H.Lev. & 가시복분자딸기 \\
Leguminosae & Indigofera koreana Ohwi & 좀땅비싸리 \\
& Lespedeza maritima Nakai & 해변싸리 \\
Labiatae & Isodon inflexus var. canescens (Nakai) Kudo & 털산박하 \\
Liliaceae & Hemerocallis hakuunensis Nakai & 백 운산원추리 \\
Umbelliferae & Peucedanum chujaense K. Kim, S.-H. Oh, C.-S. Kim \& C.-W. Park & 갈기기름나물 \\
\hline
\end{tabular}
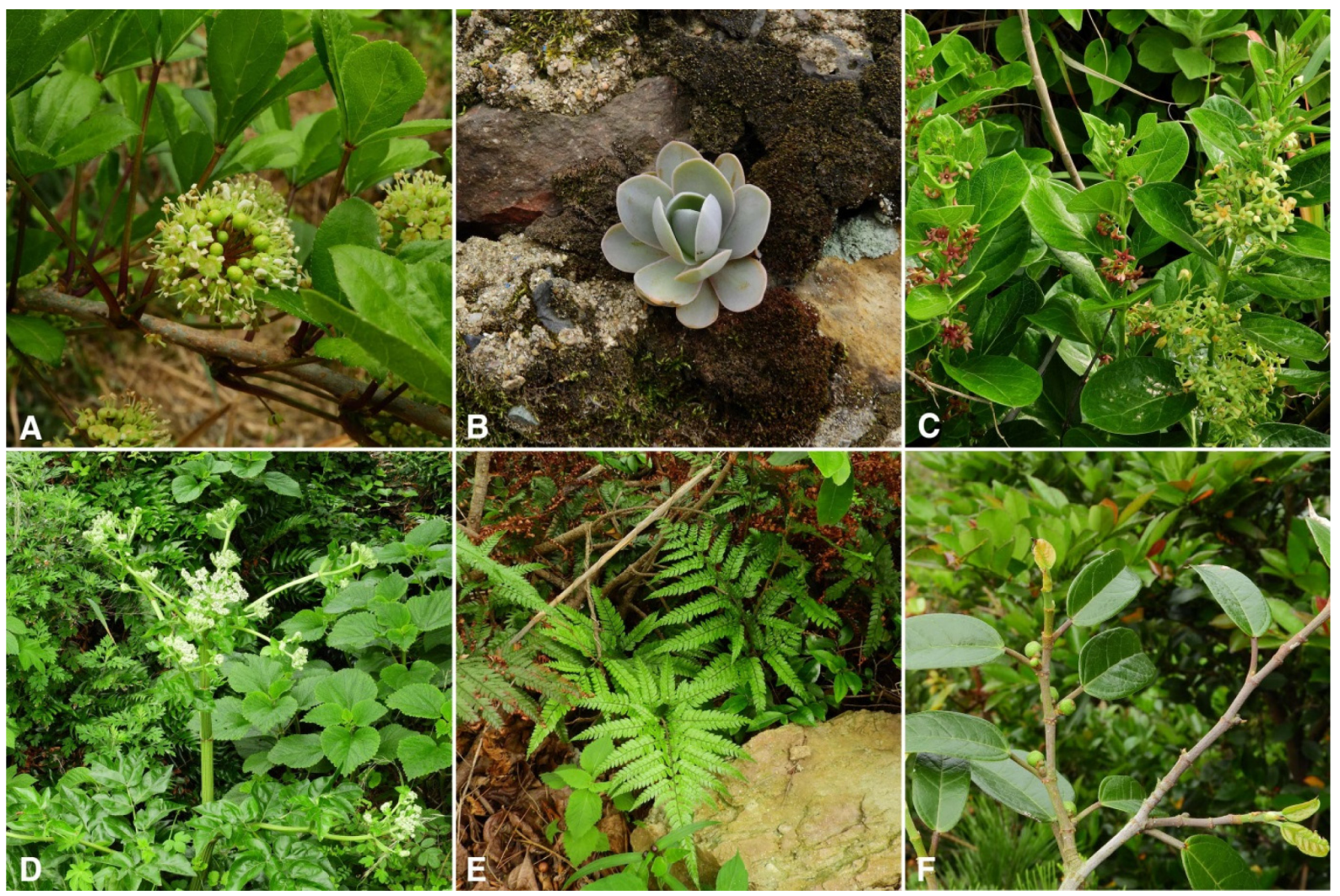

Fig. 2. The photograph of plants in Chujado Island. A. Eleutherococcus gracilistylus (W. W. Sm.) S. Y. Hu. B. Orostachys iwarenge (Makino) H. Hara. C. Cynanchum japonicum (C. Morren \& Decne.) Hemsl. D. Angelica japonica A. Gray. E. Arachniodes aristata (G. Forst.) Tindale. F. Ficus oxyphylla Miq. ex Zoll.

\section{특산식물}

본 연구에서 확인된 특산식물은 검팽나무(Celtis choseniana Nakai) 등 총 7 분류군이다(Table 3). 이는 우리나라 특산식 물 360분류군(Chung et al., 2017)의 1.9\%에 해당한다(Table 3). 검팽나무는 상추자도 큰산의 북서쪽 사면의 숲 가장자 리에 소나무와 함께 자라고 있었으며, 백운산원추리는 나 바론절벽에서 큰산으로 가는 등산로에서 단독개체로 산 발적으로 출현하고 있었다. 2019년 신종으로 새롭게 기재 된 갈기기름나물(Peucedanum chujaense K. Kim, S.-H. Oh,
C.-S. Kim \& C.-W. Park) (Kim et al., 2019)이 하추자도의 돈 대산과 추석산 일대에서 확인되었으며, 비름과에 속하는 미기록종 Achyranthes sp. (남방쇠무릎(가칭))가 발견되어 추후 추가적인 조사를 수행하고자 한다.

\section{희귀식물}

추자도에서 확인된 희귀식물(Korea National Arboretum, 2008)은 총 8 분류군으로 560 분류군의 $1.4 \%$ 에 해당된다. 이 중 위기종(endangered species, EN)은 눈향나 
무(Juniperus chinensis var. sargentii A. Henry)를 포함하여 4 분류군이었으며, 취약종(vulnerable, VU)은 연화바위솔 (Orostachys iwareng (Makino) Hara) 등 4분류군이 조사되 었다(Table 4). 추자도의 섬오갈피나무(Eleutherococcus gracilistylus (W. W. Sm.) S. Y. Hu) (Fig. 2A)는 상추자도의 봉굴레산 남동사면에서, 연화바위솔(Orostachys iwarenge (Makino) Hara) (Fig. 2B)은 나바론 절벽과 봉굴레산의 다 소 습한 곳에서 관찰되었다. 덩굴민백미꽃(Cynanchum japonicum Morr. \& Decne.) (Fig. 2C)은 나바론절벽에서 추 자등대로 가는 임도 주변에 갯메꽃, 돌가시나무, 해국 등 과 혼생하고 있었다.

\section{식물구계학적 특정식물}

환경부지정 식물구계학적 특정식물종은 Hong et al. (2014)에 의해 총 45 분류군이 밝혀진 바 있으며, 본 조사에 서는 55 과 90 속 102 종 7 변종 총 109 분류군이 확인되었고, 추자도에서 확인된 전체 분류군의 $20.1 \%$ 에 해당되었다. $\mathrm{V}$ 등급종은 눈향나무(J. chinensis var. sargentii A. Henry) 1 분류군이며, IV등급종은 갯강활(Angelica japonica A.Gray) (Fig. 2D)을 포함한 13분류군이고, III등급종은 가는쇠고사 리(Arachniodes aristata (G. Forst.) Tindale) (Fig. 2E)를 비롯 하여 44분류군이다. II등급종은 모람(Ficus oxyphylla Miq. ex Zoll.) (Fig. 2F) 등 9분류군이고, I등급종은 실고사리

Table 4. The list of rare plants in Chujado Island designated by Korea Forest Service.

\begin{tabular}{|c|c|c|c|c|}
\hline No. & Family name & Scientific name & Korean name & Criteria \\
\hline 1 & Cupressaceae & Juniperus chinensis var. sargentii A.Henry & 눈향나무 & \multirow{4}{*}{$\mathrm{EN}$} \\
\hline 2 & Araliaceae & Eleutherococcus gracilistylus (W.W.Sm.) S.Y.Hu & 섬오갈피나무 & \\
\hline 3 & Primulaceae & Lysimachia barystachys Bunge & 까치수염 & \\
\hline 4 & Asclepiadaceae & Cynanchum japonicum Morr. \& Decne. & 덩굴민백미꽃 & \\
\hline 5 & Crassulaceae & Orostachys iwarenge (Makino) Hara & 연화바위솔 & \multirow{4}{*}{ VU } \\
\hline 6 & Umbelliferae & Bupleurum falcatum $\mathrm{L}$. & 시호 & \\
\hline 7 & Myrsinaceae & Ardisia crenata Sims & 백 량금 & \\
\hline 8 & Orobanchaceae & Aeginetia indica L. & 야고 & \\
\hline
\end{tabular}

EN, endangered; VU, vulnerable.

Table 5. The list of Chujado Island of floristic regional indicator plants specially designated by the Ministry of Environment.

\begin{tabular}{|c|c|c|}
\hline Grade & Taxa & Taxa \\
\hline $\mathrm{V}$ & Juniperus chinensis var. sargentii A. Henry 눈향나무 & \\
\hline \multirow[t]{7}{*}{ IV } & Thelypteris angustifrons (Miq.) Ching 탐라사다리고사리 & Angelica japonica A. Gray 갯강활 \\
\hline & Thuja orientalis L. 측백나무 & Cynanchum japonicum Morr. \& Decne. 덩굴민백미꽃 \\
\hline & Piper kadsura (Choisy) Ohwi 후추등 & Aeginetia indica L. 야고 \\
\hline & Ternstroemia gymnanthera (Wight \& Arn.) Sprague 후피향나무 & Lilium leichtlinii var. maximowiczii (Regel) Baker 중나리 \\
\hline & Rubus nishimuranus Koidz. 제주산딸기 & Pinellia tripartita (Blume) Schott 대반하 \\
\hline & Rubus ribisoideus Matsum. 섬딸기 & Cladium chinense Nees 층층고랭이 \\
\hline & Eleutherococcus gracilistylus (W.W.Sm.) S.Y.Hu 섬오갈피나무 & \\
\hline \multirow[t]{10}{*}{ III } & Selaginella involvens (Sw.) Spring 바위손 & Rubus hirsutus Thunb 장딸기 \\
\hline & Sceptridium japonicum (Prantl) Lyon 산꽃고사리삼 & Rubus schizostylus H.Lev. 가시복분자딸기 \\
\hline & Sphenomeris chinensis (L.) Maxon 바위고사리 & Poncirus trifoliata Raf. 탱자나무 \\
\hline & Onychium japonicum (Thunb.) Kunze 선바위고사리 & Melia azedarach L. 멀구슬나무 \\
\hline & Asplenium sarelii Hook. 돌담고사리 & Acer palmatum Thunb. 단풍나무 \\
\hline & Arachniodes aristata (G. Forst.) Tindale 가는쇠고사리 & Ilex integra Thunb. 감탕나무 \\
\hline & Dryopteris championi (Benth.) C. Chr. ex Ching 제주지네고사리 & Sageretia theezans (L.) Brongn. 상동나무 \\
\hline & Thelypteris acuminata (Houtt.) C. V. Morton 별고사리 & Elaeagnus glabra Thunb. 보리장나무 \\
\hline & Lepisorus onoei (Franch. \& Sav.) Ching 애기일엽초 & Trichosanthes kirilowii var. japonica Kitam. 노랑하늘타리 \\
\hline & Castanopsis sieboldii (Makino) Hatus. 구실잣밤나무 & Dendropanax morbiferus H. Lev. 황칠나무 \\
\hline
\end{tabular}


Table 5. Continued.

\begin{tabular}{|c|c|c|}
\hline Grade & Taxa & Taxa \\
\hline \multirow[t]{12}{*}{ III } & Quercus glauca Thunb. 종가시나무 & Fatsia japonica (Thunb.) Decne. \& Planch. 팔손이 \\
\hline & Ficus erecta Thunb. 천선과나무 & Centella asiatica (L.) Urb. 병풀 \\
\hline & Ficus erecta var. sieboldii (Miq.) King 좁은잎천선과나무 & Ardisia crenata Sims 백량금 \\
\hline & Ficus thunbergii Maxim. 왕모람 & Hedyotis biflora var. parvifolia Hook. \& Arn. 낚시돌풀 \\
\hline & Kadsura japonica (L.) Dunal 남오미자 & Calystegia dahuricus (Herb.) Choisy 선메꽃 \\
\hline & Litsea japonica (Thunb.) Juss 까마귀쪽나무 & Dichondra repens Forster 아욱메풀 \\
\hline & Stauntonia hexaphylla (Thunb.) Decne. 멀꿀 & Wahlenbergia marginata (Thunb.) A. DC. 애기도라지 \\
\hline & Sinomenium acutum (Thunb.) Rehder \& E. H. Wilson 방기 & Farfugium japonicum (L.) Kitam. 털머위 \\
\hline & Eurya emarginata (Thunb.) Makino 우묵사스레피 & Sigesbeckia orientalis L. 제주진득찰 \\
\hline & Pittosporum tobira (Thunb.) W. T. Aiton 돈나무 & Asparagus cochinchinensis (Lour.) Merr. 천문동 \\
\hline & Rhaphiolepis indica var. umbellata (Thunb.) Ohashi 다정큼나무 & Ophiopogon jaburan (Kunth) Lodd. 맥문아재비 \\
\hline & Rubus buergeri Miq. 겨울딸기 & Arisaema thunbergii Blume 무늬천남성 \\
\hline \multirow[t]{5}{*}{ II } & Ficus oxyphylla Miq. ex Zoll. 모람 & Vitex rotundifolia L.f. 순비기나무 \\
\hline & Indigofera pseudotinctoria Matsum. 낭아초 & Plantago camtschatica Cham. ex Link 개질경이 \\
\hline & Phellodendron amurense Rupr. 황벽나무 & Arisaema heterophyllum Blume 두루미천남성 \\
\hline & Peucedanum japonicum Thunb. 갯기름나물 & Epipactis thunbergii A.Gray 닭의난초 \\
\hline & Argusia sibirica (L.) Dandy 모래지치 & \\
\hline \multirow[t]{21}{*}{ I } & Lygodium japonicum (Thunb.) Sw. 실고사리 & Rhamnella franguloides (Maxim.) Weberb. 까마귀베개 \\
\hline & Pteris multifida Poir. 봉의꼬리 & Cayratia japonica (Thunb.) Gagnep. 거지덩굴 \\
\hline & Dryopteris erythrosora (D. C. Eaton) Kuntze 홍지 네고사리 & Grewia parviflora Bunge 장구밤나무 \\
\hline & Lemmaphyllum microphyllum C. Pres1 콩짜개덩굴 & Elaeagnus macrophylla Thunb. 보리밥나무 \\
\hline & Celtis biondii Pamp. 폭나무 & Gynostemma pentaphyllum (Thunb.) Makino 돌외 \\
\hline & Celtis choseniana Nakai 검팽나무 & Hedera rhombea (Miq.) Bean 송악 \\
\hline & Tetragonia tetragonoides (Pall.) Kuntze 번행초 & Ardisia japonica (Thunb.) Blume 자금우 \\
\hline & Silene aprica var. oldhamiana (Miq.) C. Y. $\mathrm{Wu}$ 갯장구채 & Anagallis arvensis var. caerulea (L.) Gouan 뚜껑별꽃 \\
\hline & Machilus thunbergii Siebold \& Zucc. 후박나무 & Lysimachia barystachys Bunge 까치수염 \\
\hline & Semiaquilegia adoxoides (DC.) Makino 개구리발톱 & Lysimachia mauritiana Lam. 갯까치수염 \\
\hline & Camellia japonica L. 동백나무 & Symplocos tanakana Nakai 검노린재나무 \\
\hline & Eurya japonica Thunb. 사스레피나무 & Ligustrum japonicum Thunb. 광나무 \\
\hline & Sanguisorba tenuifolia Fisch. ex Link 가는오이풀 & Calystegia soldanella (L.) Roem. \& Schultb. 갯메꽃 \\
\hline & Euphorbia esula L. 흰대극 & Lithospermum zollingeri A. DC. 반디지치 \\
\hline & Euphorbia pekinensis Rupr. 대극 & Caryopteris incana (Thunb.) Miq. 층꽃나무 \\
\hline & Mallotus japonicus (Thunb.) Muell. Arg. 예덕나무 & Artemisia rubripes Nakai 덤불쑥 \\
\hline & Orixa japonica Thunb. 상산 & Aster sphathulifolius Maxim. 해국 \\
\hline & Zanthoxylum planispinum Siebold \& Zucc. 개산초 & Cynodon dactylon (L.) Pers. 우산잔디 \\
\hline & Euonymus fortunei (Turcz.) Hand.-Mazz. 좀사철나무 & Phacelurus latifolius (Steud.) Ohwi 모새달 \\
\hline & Euonymus japonicus Thunb. 사철나무 & Arisaema ringens (Thunb.) Schott 큰천남성 \\
\hline & Euscaphis japonica (Thunb.) Kanitz 말오줌때 & Carex lenta D. Don 줄사초 \\
\hline
\end{tabular}


(Lygodium japonicum (Thunb.) Sw.) 등 42분류군으로 나타 났다(Table 5).

\section{귀화식물}

추자도는 지리학적으로 외래식물에 취약한 도서지역 으로 본 섬에서 확인된 생태계교란식물인 미국쑥부쟁이 (Aster pilosus Willd.)와 서양금혼초(Hypochaeris radicata L.), 애기수영(Rumex acetosella L.) 3분류군을 비롯하여, 세
열미국쥐손이(Geranium dissectum L.) 등을 포함한 총 62분 류군의 귀화식물을 확인하였다(Table 6). 이는 한반도에 분포하는 귀화식물 321 분류군(Park, 1995, 2005)의 $19.3 \%$ 에 해당되며, 귀화율은 $10.6 \%$ 으로 산출되었다. 추자도 인 접 지역 7 개 도서지역과 비교한 결과, 귀화식물의 종 수는 추자도가 총 62 분류군으로 가장 많았으며, 생일도 35 분류 군(Son et al., 2013), 영산도 32분류군(Lee, 2016), 장도는 24 분류군(Oh and Beon, 2011) 등으로 추자도가 귀화식물의

Table 6. The list of invasive species in Chujado Island.

\begin{tabular}{|c|c|c|c|}
\hline Family & Taxa & Family & Taxa \\
\hline \multirow[t]{2}{*}{ Polygonaceae } & Rumex acetosella L. 애기수영 & Scrophulariaceae & Veronica hederaefolia L. 눈개불알풀 \\
\hline & Rumex crispus L. 소리쟁이 & & Veronica persica Poir. 큰개불알풀 \\
\hline Phytolaccaceae & Phytolacca americana L. 미국자리공 & Plantaginaceae & Plantago lanceolata L. 창질경이 \\
\hline \multirow[t]{2}{*}{ Caryophyllaceae } & Cerastium glomeratum Thuill. 유럽점나도나물 & Compositae & Aster pilosus Willd. 미국쑥부쟁이 \\
\hline & Silene gallica L. 양장구채 & & Aster subulatus Michx. 비짜루국화 \\
\hline \multirow[t]{3}{*}{ Chenopodiaceae } & Chenopodium album L. 흰명아주 & & Bidens pilosa L. 울산도깨비바늘 \\
\hline & Chenopodium ambrosioides L. 양명아주 & & Conyza canadensis (L.) Cronquist 망초 \\
\hline & Chenopodium glaucum L. 취명아주 & & Conyza sumatrensis E. Walker 큰망초 \\
\hline Amaranthaceae & Amaranthus viridis L. 청비름 & & Cosmos bipinnatus Cav. 코스모스 \\
\hline Papaveraceae & Papaver rhoeas L. 개양귀비 & & Erechtites hieracifolia Raf. 붉은서나물 \\
\hline \multirow[t]{5}{*}{ Cruciferae } & Brassica juncea (L.) Czern. 갓 & & Erigeron annuus (L.) Pers. 개망초 \\
\hline & Coronopus didymus (L.) Sm. 냄새냉이 & & Gnaphalium calviceps Fernald 선풀솜나물 \\
\hline & Lepidium virginicum L. 콩다닥냉이 & & Gnaphalium purpureum L. 자주풀솜나물 \\
\hline & Sisymbrium officinale var. leiocarpum DC. & & Hypochaeris radicata L. 서양금혼초 \\
\hline & 민유럽장대 & & Senecio vulgaris L. 개쑥갓 \\
\hline \multirow[t]{8}{*}{ Leguminosae } & Medicago lupulina L. 잔개자리 & & Sonchus asper (L.) Hill 큰방가지똥 \\
\hline & Medicago polymorpha L. 개자리 & & Sonchus oleraceus L. 방가지똥 \\
\hline & Robinia pseudoacacia L. 아까시나무 & & Taraxacum officinale Weber 서양민들레 \\
\hline & Trifolium campestre Schreb. 노랑토끼풀 & Amaryllidaceae & Zephyranthes candida (Lindl.) Herb. \\
\hline & Trifolium dubium Sibth. 애기노랑토끼풀 & & 흰꽃나도사프란 \\
\hline & Trifolium incarnatum L. 진홍토끼풀 & Iridaceae & Sisyrinchium angustifolium Mill. 등심붓꽃 \\
\hline & Trifolium pratense L. 붉은토끼풀 & Gramineae & Avena fatua L. 메귀리 \\
\hline & Trifolium repens L. 토끼풀 & & Briza minor L. 방울새풀 \\
\hline Oxalidaceae & Oxalis articulata Sabigny 덩이괭이밥 & & Bromus unioloides H.B. \& K. 큰이삭풀 \\
\hline Geraniaceae & Geranium dissectum L. 세열미국쥐손이 & & Dactylis glomerata L. 오리새 \\
\hline Euphorbiaceae & Euphorbia supina Raf. 애기땅빈대 & & Festuca arundinacea Schreb. 큰김의털 \\
\hline Malvaceae & Malva neglecta Wallr. 난쟁이아욱 & & Festuca myuros L. 들묵새 \\
\hline Umbelliferae & Anthriscus caucalis M. Bieb. 유럽전호 & & Lolium multiflorum Lam. 쥐보리 \\
\hline \multirow[t]{2}{*}{ Convolvulaceae } & Ipomoea purpurea Roth 둥근잎나팔꽃 & & Lolium perenne L. 호밀풀 \\
\hline & Quamoclit pennata (Desr.) Bojer 유홍초 & & Parapholis incurva (L.) C. E. Hubb. 뿔이삭풀 \\
\hline Solanaceae & Datura stramonium L. 흰독말풀 & & Paspalum dilatatum Poir. 큰참새피 \\
\hline Scrophulariaceae & Veronica arvensis L. 선개불알풀 & & Sporobolus fertilis (Steud.) Clayton 쥐꼬리새풀 \\
\hline
\end{tabular}




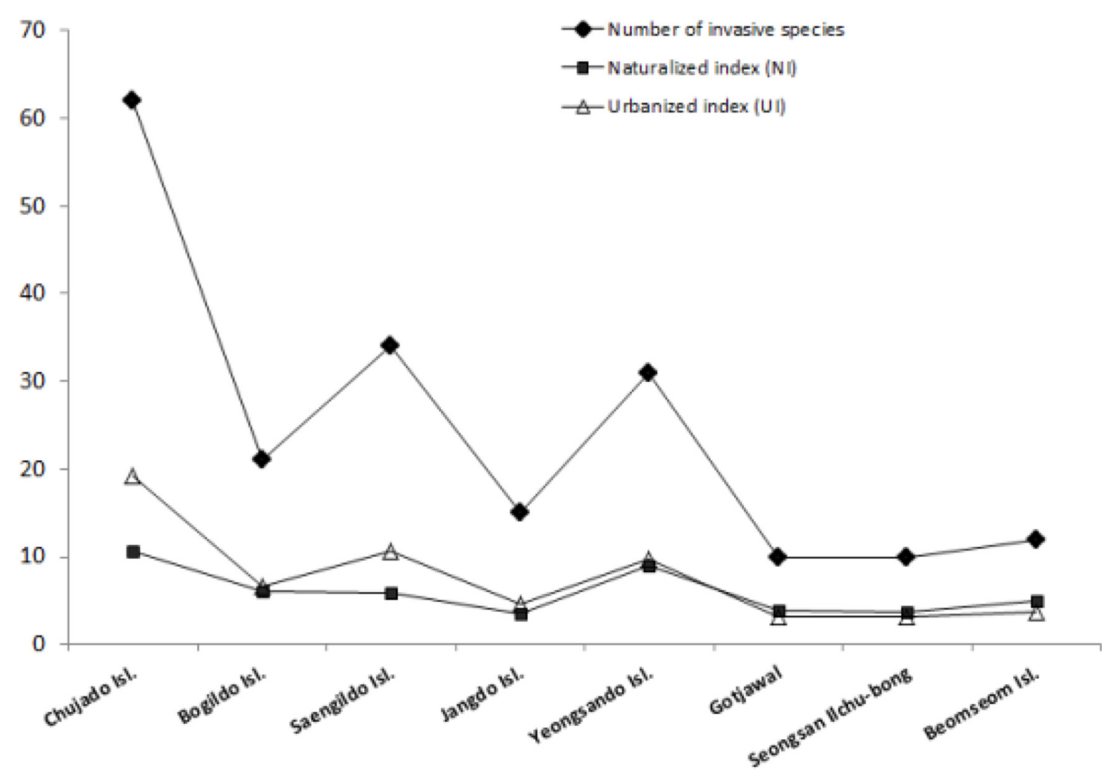

Fig. 3. The comparison of the number of invasive species, NI and UI for the 7 site near Chujado Island. From left, Chujado Isl. in Jeju, Bogildo Isl. in Cheonnam, Saengildo Isl. in Cheonnam, Jangdo Isl. in Cheonnam, Yeongsando Isl. in Cheonnam, Hanseong Gotjawal in Jeju, Seongsanilchul-bong in Jeju, Beomseom Isl. in Jeju.

Table 7. The comparison of the similarity indices (coefficient of community: \%) for the 7 site near Chujado Island.

\begin{tabular}{|c|c|c|c|c|c|c|c|}
\hline Site & 1 & 2 & 3 & 4 & 5 & 6 & 7 \\
\hline 2 & 0.38461538 & & & & & & \\
\hline 3 & 0.51657941 & 0.4946 & & & & & \\
\hline 4 & 0.474059 & 0.4753 & 0.5521 & & & & \\
\hline 5 & 0.49559471 & 0.446 & 0.5472 & 0.5747 & & & \\
\hline 6 & 0.36185819 & 0.4231 & 0.3634 & 0.3829 & 0.3808 & & \\
\hline 7 & 0.46060606 & 0.323 & 0.3557 & 0.3761 & 0.7561 & 0.7332 & \\
\hline 8 & 0.41935484 & 0.3266 & 0.2337 & 0.3568 & 0.4054 & 0.4519 & 0.87623 \\
\hline
\end{tabular}

1, Chujado Isl.; 2, Bogildo Isl. (Oh and Beon, 2011); 3, Saengildo Isl. (Lee, 2005); 4, Jangdo Isl. (Son et al., 2013); 5, Yeongsando Isl. (Lee, 2016); 6, Hangyeng Gotjawal (Park, 2019); 7, Seongsan Ilchul-bong (Kang, 2011); 8, Beomseom Isl. (Kim et al., 2005).

수, 귀화율(naturalized index, NI), 도시화율(urbanized index, UI) 모두 가장 높게 조사되었다(Fig. 3). 추자도는 주변 인 접 도서에 비하여 섬의 규모가 크지 않으나 특산종과 식물 구계학적특정종의 수가 상대적으로 높은 것은(Tables 4, 5) 자연상태가 비교적 잘 보존되어 있음을 보여준다. 그러나, 귀화율과 도시화율도 크게 나타나(Fig. 3) 관광, 어업 등 인 위적인 요소들의 영향 또한 심하게 받았음을 알 수 있다.

\section{식물상 유사도}

추자도는 지리적으로 전라도와 제주도 해상의 중간에 위치하고 있어 식물분포학적 측면에서도 매우 밀접한 유 연관계가 있을 것으로 추정된다. 따라서 추자도와 전남, 제 주도에 인접한 도서 지역인 보길도(Oh and Beon, 2011), 생일 도(Lee, 2005), 장도(Son et al., 2013), 영산도(Lee, 2016), 범섬
(Kim et al., 2005)과 제주도 본섬의 한경 곶자왈(Park, 2019), 성산일출봉(Kim, 2011) 총 7개지 지역에 출현하는 식물종의 유사도(Sørensen's Coefficients for community similarity: CCs) 를 비교 분석하였다(Table 7).

비교 대상지역과 추자도의 식물상 유사도를 보면, 전남 생일도가 $51.7 \%(0.51657941)$ 로 가장 높았으며, 제주 한경 곶자왈이 $36.2 \%(0.36185819)$ 로 가장 낮게 산출되었다. 거 리상 가장 인접한 보길도 $(38.4 \%, 0.38461538)$ 보다 생일도 가 식물상 유사도가 가장 높은데, 이는 보길도 식물상조사 (Oh and Beon, 2011)가 적자봉을 중심으로 이루어져 해안 및 마을 주변 등 섬 전체의 식물상을 반영하지 못했기 때 문이라 판단된다. 전체 7 개 지역에서는 제주 성산일출봉 과 범섬의 유사도가 가장 높고 $(87.6 \%, 0.87623)$, 전남 생일 도와 제주 범섬이 가장 낮게 $(22.4 \%, 0.2237)$ 나타났다. 유사 
도 지수가 0.2 이하이면 이질적인 군락, 0.8 이상이면 동일 군락, 1 이면 완전 같은 군락, 0 이면 완전히 다른 군락으로 해석할 수 있으며(Cox, 1972), 0.5 이상이면 통례적으로 차 이가 없는 군락으로(Yun and Hong, 2000) 처리되기도 한다.

ORCID: Eun-Mi SUN https://orcid.org/0000-0001-7691-8094; Yu Chul PARK https://orcid.org/0000-0003-2747-6044; KangHyup LEE https://orcid.org/0000-0002-7189-3235; Kuk Hwa SONG https://orcid.org/0000-0002-8433-1506; Dong Chan SON https://orcid.org/0000-0002-6773-0580

\section{Acknowledgments}

This study was supported by the project "KNA1-1-18, 153" funded by the Korea National Arboretum.

\section{Conflict of Interest}

The authors declare that there are no conflicts of interest.

\section{Literature Cited}

Chung, G. Y., K. S. Chang, J.-M. Chung, H. J. Choi, W.-K. Paik and J.-O. Hyun. 2017. A checklist of endemic plants on the Korean Peninsula. Korean Journal of Plant Taxonomy 47: 264-288. (in Korean)

Cox, C. W. 1972. Laboratory Manual of General Ecology (No. 574.5 C6). William C Brown Co. Publishers, Dubuque, IW, $195 \mathrm{pp}$.

Hong, J.-K., S.-Y. Jung, S.-J. Ji, S.-H. Park, J.-C. Yang and K.-C. Jang. 2014. The vascular plants in Chujado, Korea. Korean Society of Forest Science, Scholarship presentation collection of dissertations. Korean Forestry Society, Seoul, 54 pp. (in Korean)

Jung, Y. H., K. J. Kim and B. Y. Sun. 1986. Degree of green naturality and flora of Chuja archipelago Island. Natural Facts Report 5: 171-212. (in Korean)

Kang, S. J. 2011. Vegetation and flora of Seongsan Ilchulbong, Jeju Island, Korea. M.S. thesis. Jeju National University, Jeju, 40 pp. (in Korean)

Kim, C. S., G. P. Song, M. O. Moon, K. M. Song, J. Kim and E. J. Lee. 2005. The flora of Beonseom Island, Jeju-do. Korean Journal of Plant Resources 18: 285-301. (in Korean)

Kim, K., C.-S. Kim, S.-H. Oh and C.-W. Park. 2019. A new species of Peucedanum (Apiaceae) from Korea. Phytotaxa 393: 75-83.

Ko, P. Y., G. E. Lee, J. H. Choa, K. M. Song, G. P. Song and Y. C. Jeun. 2013. Ecological survey of plants growing in the unin- habited islets surrounding Jeju and Chuja Island. Journal of Asian Agriculture and Biotechnology 29: 53-81.

Korea Meteotological Administration. 2019. Meteorological information. Retrived Sep 10, 2019, available from https:// data.kma.go.kr.

Korea National Arboretum. 2008. Rare Plants Data Book in Korea. Korea National Arboretum, Pocheon, 412 pp.

Korea National Arboretum. 2017. Checklist of Vascular Plants in Korea. Korea National Arboretum, Pocheon, 1,000 pp.

Lee, H. 2016. Floristic study of Yeongsando in Dadohaehaesang National Park, Korea. Journal of National Park Research 7: 129-146. (in Korean)

Lee, T. B. 1969. Flora of Chuja archipelago Island, Biological Report of Chuja Island. Culture and Public Information, the Office of Cultural Properties, Seoul. Pp. 22-38. (in Korean)

Lee, W.-T. and Y. J. Yim. 1978. Studies on the distribution of vascular plants in the Korean Peninsula. Korean Journal of Plant Taxonomy 8: 1-33. (in Korean)

Lee, W. T. 1996. Coloured Standard Illustrations of Korean Plants. Academy Publishing Co., Seoul, 624 pp. (in Korean)

Lee, Y. H. 2005. The Flora in Saeng-il Island and the geographical distribution of ants. Ph.D. dissertation. Chonnam National University, Gwangju, 54 pp. (in Korean)

Lee, Y. N and Y. J. Oh. 1969. Plant Istribution Peculiarity of Chuja Islands in the Plant Distribution Region, Biological Report of Chuja Island. Culture and Public Information, The Office of Cultural Properties, Seoul. Pp. 39-46. (in Korean)

Lim, J. W., H. J. Lee, C. H. Kim and S. H. Kim. 1982. A taxonomical and ecological study on the flora of Chuja Island. Korean Journal of Ecology 5: 187-203.

Ministry of Environment. 2014. A Guide to the Fourth National Natural Environment Research. Ministry of Environment, Gwacheon and Incheon. Pp. 173-220. (in Korean)

Oh, H.-K. and M.-S. Beon. 2011. Characteristics type of vascular plants in Jeokjabong, Bogil Island (Jeonnam). Journal of Korean Environmental Restoration Technology 14: 25-40. (in Korean)

Park, J. H. 2019. Flora of Hangyeong Gotjawall Forest genetic resource reserve area in Jeju-do. M.S. thesis. Semyung University, Jecheon, 36 pp. (in Korean)

Park, M. K. 1969. Pteridophyta of Chuja archipelago Island. Biological report of Chuja Island. Culture and Public Information, The Office of Cultural Properties, Seoul. Pp. 47-48.

Park, S. H. 1995. Coloured Illustrated Guide to Naturalized Plant of Korea. Ilchogak, Seoul, 371 p. (in Korean)

Park, S. H. 2009. New Illustrations and Photographs of Naturalized Plants of Korea. Ilchokak, Seoul, 575 pp. (in Korean)

Son, H.-D., S.-G. Gwon, J. W. Jang, E.-M. Sun, B.-A. Kim and H.- 
T. Im. 2013. Floristic study of Jang-do (Isl.) in Korea. Journal of Species Research 2: 227-244.

Sörensen, T. 1948. A method of establishing groups of equal amplitude in plant society based on similarity of species content and its application to analyses of the vegetation on Danish commons. Kongelige Danske Videnskabernes Selskab, Biologiske Skrifter, 5: 1-34.
Yang, Y. H. 2006. Flora of Chuja Island, Chuja Island Scientific Research Report. Folklore \& Natural History Museum Jeju Special Self-Governing Province, Jeju. Pp. 36-51. (in Korean) Yun, C.-W. and S.-C. Hong. 2000. Quantitative analysis of vegetation types in Pinus densiflora for. erecta forest. The Korean Journal of Ecology 23: 281-291.

\title{
추자도(제주도) 지역의 관속식물 \\ 선은미 · 박유철 · 이강협 · 송국화 · 손동찬* \\ 국립수목원 산림생물다양성연구과, ${ }^{1}$ 국립생태원 자연환경조사팀
}

\begin{abstract}
적 요: 본 연구는 제주도 제주시 추자면에 위치한 추자도의 관속식물상을 조사하기 위해 2013년 4월부터 동년 9월, 2018년 5월부터 동년 9월까지 총 11회에 걸쳐 현지조사를 수행하였다. 조사 결과 추자도의 관속식 물은 총 97 과 311 속 481종 2아종 65 변종 10 품종의 558 분류군이 조사되었다. 한반도 특산식물은 7 분류군이며, 희 귀식물은 8 분류군이다. 식물구계학적등급종은 109 분류군이었으며, 귀화식물은 62 분류군으로 확인되었다. 추 자도와 인접한 7 개 지역과의 식물상 유사도는 생일도가 $51.7 \%$ 로 가장 높게 나타났다.
\end{abstract}

주요어: 추자도, 식물상, 식물지리학, 유사도

Appendix 1. The list of vascular plants collected from the Chujado Island.

\begin{tabular}{|c|c|c|c|}
\hline No. & Taxa & Korea name & Voucher number \\
\hline & Selaginellaceae 부처손과 & & \\
\hline \multirow[t]{2}{*}{1} & Selaginella involvens (Sw.) Spring & 바위손 & KHB1435967 \\
\hline & Ophioglossaceae 고사리삼과 & & \\
\hline \multirow[t]{2}{*}{2} & Sceptridium japonicum (Prantl) Lyon & 산꽃고사리삼 & KHB1374246 \\
\hline & Osmundaceae 고비과 & & \\
\hline \multirow[t]{2}{*}{3} & Osmunda japonica Thunb. & 고비 & P133431 \\
\hline & Schizaeaceae 실고사리과 & & \\
\hline \multirow[t]{2}{*}{4} & Lygodium japonicum (Thunb.) Sw. & 실고사리 & KHB1435646 \\
\hline & Pteridaceae 고사리과 & & \\
\hline 5 & Dennstaedtia hirsuta (Sw.) Mett. ex Miq. & 잔고사리 & KHB1374250 \\
\hline 6 & Pteridium aquilinum var. latiusculum (Desv.) Underw. ex Hell. & 고사리 & KHB1435534 \\
\hline 7 & Sphenomeris chinensis (L.) Maxon & 바위고사리 & KHB1436031 \\
\hline 8 & Onychium japonicum (Thunb.) Kunze & 선바위고사리 & KHB1435466 \\
\hline \multirow[t]{2}{*}{9} & Pteris multifida Poir. & 봉의꼬리 & Son 4042 \\
\hline & Aspleniaceae 꼬리고사리과 & & \\
\hline 10 & Asplenium incisum Thunb. & 꼬리고사리 & KHB1435610 \\
\hline \multirow[t]{2}{*}{11} & Asplenium sarelii Hook. & 돌담고사리 & Lee1805012 \\
\hline & Dryopteridaceae 면마과 & & \\
\hline 12 & Arachniodes aristata (G. Forst.) Tindale & 가는쇠고사리 & KHB1360582 \\
\hline
\end{tabular}


Appendix 1. Continued.

\begin{tabular}{|c|c|c|c|}
\hline 13 & Cyrtomium devexiscapulae (Koidz.) Ching & 긴잎도깨비쇠고비 & $O B-P L-180300012$ \\
\hline 14 & Cyrtomium falcatum (L. f.) C. Presl & 도깨비쇠고비 & KHB1436016 \\
\hline 15 & Cyrtomium fortunei J. Sm. & 쇠고비 & P133432 \\
\hline 16 & Dryopteris bissetiana (Baker) C. Chr. & 산족제비고사리 & KHB1440865 \\
\hline 17 & Dryopteris championi (Benth.) C. Chr. ex Ching & 제주지네고사리 & KHB1435840 \\
\hline 18 & Dryopteris chinensis (Baker) Koidz. & 가는잎족제비고사리 & KHB1435886 \\
\hline 19 & Dryopteris erythrosora (D. C. Eaton) Kuntze & 홍지네고사리 & KHB1450936 \\
\hline 20 & Dryopteris hikonensis (H. Ito) Nakaike & 큰족제비고사리 & KHB1360591 \\
\hline 21 & Dryopteris lacera (Thunb.) Kuntze & 비늘고사리 & KHB1435375 \\
\hline 22 & Dryopteris sacrosanta Koidz. & 애기족제비고사리 & KHB1440910 \\
\hline 23 & Dryopteris uniformis (Makino) Makino & 곰비늘고사리 & KHB1360585 \\
\hline 24 & Polystichum polyblepharum (Roem. ex Kunze) C.Presl & 나도히초미 & Hong1010980 \\
\hline 25 & Thelypteris acuminata (Houtt.) C. V. Morton & 별고사리 & KHB1360579 \\
\hline 26 & Thelypteris angustifrons (Miq.) Ching & 탐라사다리고사리 & KHB1435833 \\
\hline 27 & Thelypteris decursivepinnata (H. C. Hall) Ching & 설설고사리 & KHB1440833 \\
\hline 28 & Thelypteris glanduligera (Kunze) Ching & 사다리고사리 & KHB1436627 \\
\hline 29 & Deparia $x$ angustatum (Nakai) Nakaike & 개좀진고사리 & Son 4031 \\
\hline 30 & Deparia japonica (Thunb.) M. Kato & 진고사리 & KHB1440981 \\
\hline \multirow[t]{2}{*}{31} & Deparia lasiopteris (Kunze) Nakaike & 큰진고사리 & Son3798 \\
\hline & Polypodiaceae 고란초과 & & \\
\hline 32 & Lemmaphyllum microphyllum C. Presl & 콩짜개덩굴 & KHB1435535 \\
\hline 33 & Lepisorus onoei (Franch. \& Sav.) Ching & 애기일엽초 & KHB1436647 \\
\hline \multirow[t]{2}{*}{34} & Lepisorus thunbergianus (Kaulf.) Ching & 일엽초 & KHB1440864 \\
\hline & Pinaceae 소나무과 & & \\
\hline 35 & Pinus densiflora Siebold \& Zucc. & 소나무 & Son0601 \\
\hline \multirow[t]{2}{*}{36} & Pinus thunbergii Parl. & 곰솔 & KHB1435966 \\
\hline & Cupressaceae 측백나무과 & & \\
\hline 37 & Juniperus chinensis var. sargentii A. Henry & 눈향나무 & KHB1435863 \\
\hline \multirow[t]{2}{*}{38} & Thuja orientalis L. & 측백나무 & Son0503 \\
\hline & Betulaceae 자작나무과 & & \\
\hline \multirow[t]{2}{*}{39} & Alnus firma Siebold \& Zucc. & 사방오리 & KHB1436058 \\
\hline & Fagaceae 참나무과 & & \\
\hline 40 & Castanopsis sieboldii (Makino) Hatus. & 구실잣밤나무 & $O B-P L-180300024$ \\
\hline 41 & Quercus acutissima Carruth. & 상수리나무 & KHB1451047 \\
\hline 42 & Quercus dentata Thunb. & 떡갈나무 & KHB1363165 \\
\hline \multirow[t]{2}{*}{43} & Quercus glauca Thunb. & 종가시나무 & Son0555 \\
\hline & Ulmaceae 느릅나무과 & & \\
\hline 44 & Celtis biondii Pamp. & 폭나무 & Son0779 \\
\hline 45 & Celtis choseniana Nakai & 검팽나무 & $O B-P L-180300027$ \\
\hline 46 & Celtis sinensis Pers. & 팽나무 & KHB1374416 \\
\hline 47 & Zelkova serrata (Thunb.) Makino & 느티나무 & $P 130151$ \\
\hline
\end{tabular}


Appendix 1. Continued.

\begin{tabular}{|c|c|c|c|}
\hline & Moraceae 뽕나무과 & & \\
\hline 48 & Broussonetia papyrifera $($ L.) L'Her. ex Vent. & 꾸지나무 & KHB1363282 \\
\hline 49 & Cudrania tricuspidata (Carr.) Bureau ex Lavallee & 꾸지뽕나무 & P133335 \\
\hline 50 & Ficus carica $\mathrm{L}$. & 무화과나무 & Son3632 \\
\hline 51 & Ficus erecta Thunb. & 천선과나무 & KHB1436014 \\
\hline 52 & Ficus erecta var. sieboldii (Miq.) King & 좁은잎천선과나무 & KHB1436586 \\
\hline 53 & Ficus oxyphylla Miq. ex Zoll. & 모람 & KHB1360321 \\
\hline 54 & Ficus thunbergii Maxim. & 왕모람 & KHB1369460 \\
\hline \multirow[t]{2}{*}{55} & Morus bombycis Koidz. & 산뽕나무 & KHB1435973 \\
\hline & Cannabaceae 삼과 & & \\
\hline \multirow[t]{2}{*}{56} & Humulus japonicus Sieboid \& Zucc. & 환삼덩굴 & KHB1451042 \\
\hline & Urticaceae 쐐기풀과 & & \\
\hline 57 & Boehmeria longispica Steud. & 왜모시풀 & KHB1435424 \\
\hline 58 & Boehmeria nivea (L.) Gaudich. & 모시풀 & KHB1435423 \\
\hline 59 & Boehmeria nivea var. nipononivea (Koidz.) W. T. Wang & 섬모시풀 & KHB1436520 \\
\hline 60 & Boehmeria pannosa Nakai \& Satake & 왕모시풀 & KHB1435581 \\
\hline \multirow[t]{2}{*}{61} & Boehmeria sieboldiana Blume & 긴잎모시풀 & Lee1805007 \\
\hline & Santalaceae 단향과 & & \\
\hline \multirow[t]{2}{*}{62} & Thesium chinense Turcz. & 제비꿀 & KHB1435799 \\
\hline & Polygonaceae 마디풀과 & & \\
\hline 63 & Persicaria conspicua (Nakai) Nakai ex Mori & 꽃여뀌 & KHB1440828 \\
\hline 64 & Persicaria filiformis (Thunb.) Nakai ex Mori & 이삭여뀌 & KHB1360573 \\
\hline 65 & Persicaria japonica (Meisn.) H. Gross ex Nakai & 흰꽃여뀌 & KHB1435435 \\
\hline 66 & Persicaria lapathifolia (L.) Gray & 흰여뀌 & KHB1451020 \\
\hline 67 & Persicaria longiseta (Bruijn) Kitag. & 개여뀌 & KHB1435387 \\
\hline 68 & Persicaria posumbu var. laxiflora (Meisn.) H. Hara & 장대여뀌 & $O B-P L-180300042$ \\
\hline 69 & Persicaria senticosa (Meisn.) H. Gross ex Nakai & 며느리밑씻개 & KHB1360584 \\
\hline 70 & Persicaria thunbergii (Siebold \& Zucc.) H. Gross ex Nakai & 고마리 & KHB1435429 \\
\hline 71 & Polygonum aviculare L. & 마디풀 & KHB1435792 \\
\hline 72 & Rumex acetosa $\mathrm{L}$. & 수영 & KHB1435963 \\
\hline 73 & Rumex acetosella $\mathrm{L}$. & 애기수영 & KHB1436012 \\
\hline 74 & Rumex crispus $\mathrm{L}$. & 소리쟁이 & KHB1440969 \\
\hline 75 & Rumex japonicus Houtt. & 참소리쟁이 & KHB1435982 \\
\hline 76 & Rumex patientia $\mathrm{L}$. & 부령소리쟁이 & Son0493 \\
\hline \multirow[t]{2}{*}{77} & Phytolacca americana $\mathrm{L}$. & 미국자리공 & Son0762 \\
\hline & Nyctaginaceae 분꽃과 & & \\
\hline \multirow[t]{2}{*}{78} & Mirabilis jalapa $\mathrm{L}$. & 분꽃(식재) & KHB1451022 \\
\hline & Aizoaceae 번행초과 & & \\
\hline \multirow[t]{2}{*}{79} & Tetragonia tetragonoides (Pall.) Kuntze & 번행초 & KHB1360309 \\
\hline & Portulacaceae 쇠비름과 & & \\
\hline 80 & Portulaca oleracea L. & 쇠비름 & KHB1450926 \\
\hline
\end{tabular}


Appendix 1. Continued.

Caryophyllaceae 석죽과

81 Cerastium glomeratum Thuill.

82 Cerastium holosteoides var. hallaisanense (Nakai) Mizush.

83 Dianthus japonicus Thunb.

84 Sagina japonica (Sw.) Ohwi

85 Sagina maxima A.Gray

86 Silene aprica var. oldhamiana (Miq.) C. Y. Wu

87 Silene firma Siebold \& Zucc.

88 Silene gallica $\mathrm{L}$.

89 Spergularia marina (L.) Griseb.

90 Stellaria aquatica (L.) Scop.

91 Stellaria media (L.) Vill.

92 Stellaria neglecta (Lej.) Weihe

Chenopodiaceae 명아주과

93 Atriplex gmelinii C. A. Mey.

94 Beta vulgaris var. cicla $\mathrm{L}$.

95 Chenopodium album $\mathrm{L}$.

96 Chenopodium album var. centrorubrum Makino

97 Chenopodium ambrosioides L.

98 Chenopodium glaucum $\mathrm{L}$.

99 Kochia scoparia (L.) Schrad.

Amaranthaceae 비름과

100 Achyranthes fauriei H. Lev. \& Vaniot

101 Achyranthes japonica (Miq.) Nakai

102 Amaranthus viridis $\mathrm{L}$.

103 Achyranthes sp.

Schisandraceae 오미자과

104 Kadsura japonica (L.) Dunal

Lauraceae 녹나무과

105 Litsea japonica (Thunb.) Juss.

106 Machilus thunbergii Siebold \& Zucc.

Ranunculaceae 미나리아재비과

107 Clematis apiifolia DC.

108 Clematis terniflora DC.

109 Clematis terniflora var. mandshurica (Rupr.) Ohwi

110 Ranunculus cantoniensis DC.

111 Ranunculus chinensis Bunge

112 Ranunculus japonicus Thunb.

유럽점나도나물

점나도나물

갯패랭이꽃

개미자리

큰개미자리

갯장구채

장구채

양장구채

갯개미자리

쇠별꽃

별꽃

초록별꽃

가는갯는쟁이

근대

흰명아주

명아주

양명아주

취명아주

댑싸리

털쇠무릎

쇠무릎

청비름

남방쇠무릎

남오미자

까마귀쪽나무

후박나무

사위질빵

참으아리

으아리

털개구리미나리

젓가락나물

미나리아재비

왜젓가락나물

개구리자리

개구리발톱
KHB1436038

Son0694

KHB1419933

KHB1440808

KHB1436693

KHB1435945

Lee1805024

KHB1436067

KHB1373868

KHB1436600

KHB1436692

KHB1436001

KHB1440929

Lee1805005

KHB1435427

KHB1435626

KHB1451021

OB-PL-180300049

KHB1369496

KHB1451092

KHB1441009

KHB1040604

Lee1805009

KHB1435865

KHB1450919

Lee1805030

KHB1451048

KHB1360577

KHB1451095

KHB1360307

KHB 1363171

KHB1435960

Lee1805023

KHB1436007

KHB1436000

115 Semiaquilegia adoxoides (DC.) Makino 
Appendix 1. Continued.

Lardizabalaceae 으름덩굴과

116 Akebia quinata (Houtt.) Decne.

으름덩굴

KHB1436074

117 Stauntonia hexaphylla (Thunb.) Decne.

멀꿀

Hong1000371

Menispermaceae 새모래덩굴과

118 Cocculus trilobus (Thunb.) DC.

댕댕이덩굴

KHB1436705

119 Sinomenium acutum (Thunb.) Rehder \& E. H. Wilson

방기

$O B-P L-180300064$

Piperaceae 후추과

120 Piper kadsura (Choisy) Ohwi

Theaceae 차나무과

121 Camellia japonica $\mathrm{L}$.

122 Eurya emarginata (Thunb.) Makino

123 Eurya japonica Thunb.

124 Ternstroemia gymnanthera (Wight \& Arn.) Sprague Guttiferae 물레나물과

125 Hypericum erectum Thunb.

후추등

Hong9252

Papaveraceae 양귀비과

126 Papaver rhoeas $\mathrm{L}$.

개양귀비

Son0700

동백나무

KHB1360575

우묵사스레피

KHB1440921

사스레피나무

KHB1440900

Fumariaceae 현호색과

127 Corydalis heterocarpa Siebold \& Zucc.

염주괴불주머니

KHB 1363008

128 Corydalis incisa (Thunb.) Pers.

129 Corydalis platycarpa (Maxim.) Makino

자주괴불주머니

Hong5045

갯괴불주머니

KHB 1435964

Cruciferae 십자화과

130 Berteroella maximowiczii (Palib.) O. E. Schulz

장대냉이

KHB 1518742

131 Brassica juncea (L.) Czern.

갓

Son0582

132 Brassica napus L.

유채

KHB1435845

133 Capsella bursapastoris (L.) L.W.Medicus

냉이

KHB1436066

134 Cardamine flexuosa With.

황새냉이

KHB1435789

135 Cheiranthus cheiri L.

꽃무

Son0508

136 Coronopus didymus (L.) Sm.

냄새냉이

KHB 1435978

137 Lepidium virginicum $\mathrm{L}$.

콩다닥냉이

KHB1436584

138 Raphanus sativus var. hortensis f. raphanistroides Makino

갯무

KHB1435821

139 Sisymbrium officinale var. leiocarpum DC.

민유럽장대

Hong 1000728

Crassulaceae 돌나물과

140 Orostachys iwarenge (Makino) Hara

연화바위솔

Son 4039

141 Orostachys japonica (Maxim.) A. Berger

바위솔

KHB 1440880

142 Sedum bulbiferum Makino

말똥비름

KHB 1436665

143 Sedum japonicum Siebold ex Miq.

돌채송화

KHB 1440985

144 Sedum kamtschaticum Fisch. \& Mey.

기린초

Lee1805006

145 Sedum lepidodium Nakai

갯돌나물

Son0704

146 Sedum oryzifolium Makino

땅채송화

KHB1360589

147 Sedum sarmentosum Bunge

돌나물

KHB1360586 
Appendix 1. Continued.

Saxifragaceae 범의귀과

148 Hydrangea macrophylla (Thunb.) Ser.

수국

KHB1440862

Pittosporaceae 돈나무과

149 Pittosporum tobira (Thunb.) W.T.Aiton

돈나무

KHB1435609

Rosaceae 장미과

150 Agrimonia coreana Nakai

151 Agrimonia gorovoii Rumjantsev

산짚신나물

Son3571

152 Agrimonia pilosa Ledeb.

고로보이짚신나물

Lee1805003

153 Duchesnea indica (Andr.) Focke

짚신나물

KHB1451064

154 Geum aleppicum Jacq.

뱀딸기

KHB1435976

155 Potentilla anemonefolia Lehm.

큰뱀무

KHB 1450918

156 Potentilla chinensis Ser.

157 Potentilla freyniana Bornm.

가락지나물

Son0919

딱지꽃

KHB 1435483

158 Prunus persica (L.) Batsch

세잎양지꽃

Son0924

159 Prunus sp.

복사나무

Son0799

160 Rhaphiolepis indica var. umbellata (Thunb.) Ohashi

벚나무sp.

Son3957

161 Rosa multiflora Thunb.

다정큼나무

KHB 1450925

162 Rosa rugosa Thunb.

찔레꽃

KHB1360302

163 Rosa wichuraiana Crep. ex Franch. \& Sav.

해당화

Son 3764

164 Rubus buergeri Miq.

165 Rubus corchorifolius L.f.

돌가시나무

KHB1440945

166 Rubus crataegifolius Bunge

겨울딸기

$P 131834$

수리딸기

Son3930

167 Rubus hirsutus Thunb.

산딸기

Lee1805015

168 Rubus nishimuranus Koidz.

장딸기

KHB1435828

169 Rubus parvifolius L.

제주산딸기

Lee1805025

멍석딸기

KHB1360236

170 Rubus ribisoideus Matsum.

171 Rubus schizostylus H. Lev.

섬딸기

Son0596

172 Rubus trifidus Thunb.

가시복분자딸기

KHB1041229

거문딸기

KHB1435962

173 Sanguisorba officinalis L.

오이풀

KHB 1435590

174 Sanguisorba tenuifolia Fisch. ex Link

가는오이풀

Son0802

Leguminosae 콩과

175 Albizia julibrissin Durazz.

자귀나무

KHB1435612

176 Amphicarpaea bracteata subsp. edgeworthii (Benth.) H. Ohashi

새콩

KHB1435551

177 Chamaecrista nomame (Siebold) H.Ohashi

차풀

KHB1435526

178 Crotalaria sessiliflora $\mathrm{L}$.

활나물

Lee1805029

179 Desmodium podocarpum DC.

개도둑놈의갈고리

Son0873

180 Desmodium podocarpum var. mandshuricum Maxim.

애기도둑놈의갈고리

Son0895

181 Desmodium podocarpum var. oxyphyllum (DC.) H. Ohashi

도둑놈의갈고리

KHB 1435447

182 Dunbaria villosa (Thunb.) Makino

여우팥

KHB1450939

183 Glycine soja Siebold \& Zucc.

돌콩

KHB 1435436

184 Indigofera koreana Ohwi

좀땅비싸리

KHB1363277 


\begin{tabular}{|c|c|c|c|}
\hline 185 & Indigofera pseudotinctoria Matsum. & 낭아초 & KHB1440890 \\
\hline 186 & Kummerowia stipulacea (Maxim.) Makino & 둥근매듭풀 & KHB1435545 \\
\hline 187 & Kummerowia striata (Thunb.) Schindl. & 매듭풀 & KHB1435433 \\
\hline 188 & Lathyrus japonicus Willd. & 갯완두 & KHB1435820 \\
\hline 189 & Lespedeza bicolor Turcz. & 싸리 & Son3946 \\
\hline 190 & Lespedeza cuneata G. Don & 비수리 & КHB1435448 \\
\hline 191 & Lespedeza maritima Nakai & 해변싸리 & $O B-P L-180300108$ \\
\hline 192 & Lespedeza pilosa (Thunb.) Siebold \& Zucc. & 괭이싸리 & KHB1435643 \\
\hline 193 & Lespedeza tomentosa (Thunb.) Siebold ex Maxim. & 개싸리 & KHB1435566 \\
\hline 194 & Lespedeza virgata (Thunb.) DC. & 좀싸리 & KHB1435639 \\
\hline 195 & Lotus corniculatus var. japonica Regel & 벌노랑이 & KHB1360297 \\
\hline 196 & Medicago lupulina $\mathrm{L}$. & 잔개자리 & Son3710 \\
\hline 197 & Medicago polymorpha $\mathrm{L}$. & 개자리 & KHB1360301 \\
\hline 198 & Pisum sativum $\mathrm{L}$. & 완두 & Hong1000452 \\
\hline 199 & Pueraria lobata (Willd.) Ohwi & 칡 & KHB1435452 \\
\hline 200 & Rhynchosia volubilis Lour. & 여우콩 & KHB1435640 \\
\hline 201 & Robinia pseudoacacia $\mathrm{L}$. & 아까시나무 & KHB1363161 \\
\hline 202 & Trifolium campestre Schreb. & 노랑토끼풀 & Lee1805010 \\
\hline 203 & Trifolium dubium Sibth. & 애기노랑토끼풀 & Son3793 \\
\hline 204 & Trifolium incarnatum L. & 진홍토끼풀 & KHB1436008 \\
\hline 205 & Trifolium pratense $\mathrm{L}$. & 붉은토끼풀 & KHB1360237 \\
\hline 206 & Trifolium repens $\mathrm{L}$. & 토끼풀 & KHB1435932 \\
\hline 207 & Vicia angustifolia var. segetilis (Thuill.) K. Koch. & 살갈퀴 & KHB1435849 \\
\hline 208 & Vicia cracca $\mathrm{L}$. & 등갈퀴나물 & KHB1360299 \\
\hline 209 & Vicia hirsuta (L.) Gray & 새완두 & KHB1435984 \\
\hline 210 & Vicia japonica A. Gray & 넓은잎갈퀴 & KHB1451099 \\
\hline 211 & Vicia sepium L. & 구주갈퀴덩굴 & Lee1805004 \\
\hline \multirow[t]{2}{*}{212} & Vicia tetrasperma (L.) Schreb. & 얼치기완두 & KHB1435801 \\
\hline & Oxalidaceae 괭이밥과 & & \\
\hline 213 & Oxalis articulata Sabigny & 덩이괭이밥 & KHB1436059 \\
\hline \multirow[t]{2}{*}{214} & Oxalis corniculata $\mathrm{L}$. & 괭이밥 & KHB1435810 \\
\hline & Geraniaceae 쥐손이풀과 & & \\
\hline 215 & Geranium dissectum $\mathrm{L}$. & 세열미국쥐손이 & Lee1805018 \\
\hline 216 & Geranium krameri Franch. \& Sav. & 선이질풀 & KHB1435649 \\
\hline 217 & Geranium thunbergii Siebold \& Zucc. & 이질풀 & KHB1435525 \\
\hline \multirow[t]{2}{*}{218} & Geranium wilfordii Maxim. & 세잎쥐손이 & KHB1435458 \\
\hline & Euphorbiaceae 대극과 & & \\
\hline 219 & Acalypha australis $\mathrm{L}$. & 깨풀 & KHB1360590 \\
\hline 220 & Euphorbia esula L. & 흰대극 & KHB1435635 \\
\hline 221 & Euphorbia helioscopia L. & 등대풀 & KHB1436025 \\
\hline 222 & Euphorbia pekinensis Rupr. & 대극 & KHB1440873 \\
\hline
\end{tabular}


Appendix 1. Continued.

\begin{tabular}{|c|c|c|c|}
\hline 223 & Euphorbia supina Raf. & 애기땅빈대 & KHB1435392 \\
\hline \multirow[t]{2}{*}{224} & Mallotus japonicus (Thunb.) Muell. Arg. & 예덕나무 & KHB1436523 \\
\hline & Rutaceae 운향과 & & \\
\hline 225 & Orixa japonica Thunb. & 상산 & Hong4759 \\
\hline 226 & Phellodendron amurense Rupr. & 황벽나무 & Hong1000357 \\
\hline 227 & Poncirus trifoliata Raf. & 탱자나무 & KHB1369472 \\
\hline 228 & Zanthoxylum piperitum (L.) DC. & 초피나무 & Son0743 \\
\hline \multirow[t]{2}{*}{229} & Zanthoxylum planispinum Siebold \& Zucc. & 개산초 & KHB1435489 \\
\hline & Simaroubaceae 소태나무과 & & \\
\hline \multirow[t]{2}{*}{230} & Picrasma quassioides (D.Don) Benn. & 소태나무 & Lee1805019 \\
\hline & Meliaceae 멀구슬나무과 & & \\
\hline \multirow[t]{2}{*}{231} & Melia azedarach $\mathrm{L}$. & 멀구슬나무 & Son0527 \\
\hline & Anacardiaceae 옻나무과 & & \\
\hline 232 & Rhus javanica $\mathrm{L}$. & 붉나무 & Hong8959 \\
\hline \multirow[t]{2}{*}{233} & Rhus sylvestris Siebold \& Zucc. & 산검양옻나무 & KHB1436691 \\
\hline & Aceraceae 단풍나무과 & & \\
\hline \multirow[t]{2}{*}{234} & Acer palmatum Thunb. & 단풍나무 & Hong 1000369 \\
\hline & Aquifoliaceae 감탕나무과 & & \\
\hline \multirow[t]{2}{*}{235} & Ilex integra Thunb. & 감탕나무 & KHB1369488 \\
\hline & Celastraceae 노박덩굴과 & & \\
\hline 236 & Celastrus orbiculatus Thunb. & 노박덩굴 & KHB1360173 \\
\hline 237 & Celastrus orbiculatus var. punctatus (Thunb.) Rehder & 해변노박덩굴 & Son0798 \\
\hline 238 & Euonymus alatus f. ciliatodentatus (Franch. \& Sav.) Hiyama & 회잎나무 & KHB 1041545 \\
\hline 239 & Euonymus fortunei (Turcz.) Hand.-Mazz. & 좀사철나무 & KHB1545071 \\
\hline \multirow[t]{2}{*}{240} & Euonymus japonicus Thunb. & 사철나무 & KHB1360572 \\
\hline & Staphyleaceae 고추나무과 & & \\
\hline \multirow[t]{2}{*}{241} & Euscaphis japonica (Thunb.) Kanitz & 말오줌때 & KHB1440960 \\
\hline & Rhamnaceae 갈매나무과 & & \\
\hline 242 & Rhamnella franguloides (Maxim.) Weberb. & 까마귀베개 & Lee180532 \\
\hline \multirow[t]{2}{*}{243} & Sageretia theezans (L.) Brongn. & 상동나무 & KHB1435861 \\
\hline & Vitaceae 포도과 & & \\
\hline 244 & Ampelopsis brevipedunculata (Maxim.) Trautv. & 개머루 & KHB1435604 \\
\hline 245 & Ampelopsis brevipedunculata f. citrulloides Rehder & 가새잎개머루 & KHB1451056 \\
\hline 246 & Cayratia japonica (Thunb.) Gagnep. & 거지덩굴 & KHB1440836 \\
\hline 247 & Parthenocissus tricuspidata (Siebold \& Zucc.) Planch. & 담쟁이덩굴 & KHB1436526 \\
\hline 248 & Vitis amurensis Rupr. & 왕머루 & Hong1020620 \\
\hline 249 & Vitis ficifolia f. glabrata (Nakai) W. T. Lee & 청까마귀머루 & KHB1440902 \\
\hline 250 & Vitis ficifolia var. sinuata (Regel) H. Hara & 까마귀머루 & KHB1441014 \\
\hline \multirow[t]{2}{*}{251} & Vitis flexuosa Thunb. & 새머루 & KHB1360172 \\
\hline & Tiliaceae 피나무과 & & \\
\hline 252 & Corchoropsis tomentosa (Thunb.) Makino & 수까치깨 & KHB1435495 \\
\hline
\end{tabular}


Appendix 1. Continued.

\begin{tabular}{|c|c|c|c|}
\hline \multirow[t]{2}{*}{253} & Grewia parviflora Bunge & 장구밤나무 & KHB1545078 \\
\hline & Malvaceae 아욱과 & & \\
\hline 254 & Hibiscus syriacus $\mathrm{L}$. & 무궁화(식재) & KHB1435603 \\
\hline \multirow[t]{2}{*}{255} & Malva neglecta Wallr. & 난쟁이아욱 & KHB1435371 \\
\hline & Elaeagnaceae 보리수나무과 & & \\
\hline 256 & Elaeagnus glabra Thunb. & 보리장나무 & KHB1360592 \\
\hline 257 & Elaeagnus macrophylla Thunb. & 보리밥나무 & KHB1435971 \\
\hline \multirow[t]{2}{*}{258} & Elaeagnus umbellata Thunb. & 보리수나무 & KHB1360588 \\
\hline & Flacourtiaceae 이나무과 & & \\
\hline \multirow[t]{2}{*}{259} & Xylosma japonica (Thunb.) A. Gray ex H. Ohashi & 산유자나무 & $O B-P L-180300147$ \\
\hline & Violaceae 제비꽃과 & & \\
\hline 260 & Viola acuminata Ledeb. & 졸방제비꽃 & KHB1436641 \\
\hline 261 & Viola grypoceras A.Gray & 낚시제비꽃 & KHB1360256 \\
\hline 262 & Viola japonica Langsd. ex Ging. & 왜제비꽃 & KHB1360259 \\
\hline 263 & Viola mandshurica W.Becker & 제비꽃 & KHB1435615 \\
\hline 264 & Viola phalacrocarpa Maxim. & 털제비꽃 & KHB1436009 \\
\hline 265 & Viola phalacrocarpa f. glaberrima (W.Becker) F.Maek. ex H. Hara & 민둥제비꽃 & KHB1435993 \\
\hline \multirow[t]{2}{*}{266} & Viola verecunda A.Gray & 콩제비꽃 & KHB1436019 \\
\hline & Cucurbitaceae 박과 & & \\
\hline 267 & Gynostemma pentaphyllum (Thunb.) Makino & 돌외 & КHB1440982 \\
\hline 268 & Trichosanthes kirilowii Maxim. & 하늘타리 & KHB1436579 \\
\hline \multirow[t]{2}{*}{269} & Trichosanthes kirilowii var. japonica Kitam. & 노랑하늘타리 & KHB1436581 \\
\hline & Araliaceae 두릅나무과 & & \\
\hline 270 & Aralia elata (Miq.) Seem. & 두릅나무 & KHB1041804 \\
\hline 271 & Dendropanax morbiferus H. Lev. & 황칠나무 & Son0766 \\
\hline 272 & Eleutherococcus gracilistylus (W.W.Sm.) S. Y. Hu & 섬오갈피나무 & Son3603 \\
\hline 273 & Fatsia japonica (Thunb.) Decne. \& Planch. & 팔손이 & KHB1436047 \\
\hline \multirow[t]{2}{*}{274} & Hedera rhombea (Miq.) Bean & 송악 & КHB1360169 \\
\hline & Umbelliferae 산형과 & & \\
\hline 275 & Angelica japonica A.Gray & 갯강활 & KHB1436652 \\
\hline 276 & Anthriscus caucalis M.Bieb. & 유럽전호 & Hong1000728 \\
\hline 277 & Anthriscus sylvestris (L.) Hoffm. & 전호 & KHB1435954 \\
\hline 278 & Bupleurum falcatum $\mathrm{L}$. & 시호 & KHB 1440868 \\
\hline 279 & Centella asiatica (L.) Urb. & 병풀 & KHB1360303 \\
\hline 280 & Cnidium japonicum Miq. & 갯사상자 & KHB1435367 \\
\hline 281 & Daucus littoralis Sibth. & 갯당근 & KHB1436570 \\
\hline 282 & Hydrocotyle maritima Honda & 선피막이 & KHB 1440840 \\
\hline 283 & Oenanthe javanica (Blume) DC. & 미나리 & Son0892 \\
\hline 284 & Peucedanum japonicum Thunb. & 갯기름나물 & KHB1436585 \\
\hline 285 & Peucedanum terebinthaceum (Fisch.) Fisch. ex DC. & 기름나물 & KHB1451059 \\
\hline 286 & Torilis japonica (Houtt.) DC. & 사상자 & KHB1440966 \\
\hline
\end{tabular}


Appendix 1. Continued.

\begin{tabular}{|c|c|c|c|}
\hline 287 & Torilis scabra (Thunb.) DC. & 개사상자 & Son0588 \\
\hline \multirow[t]{2}{*}{288} & Peucedanum chujaense K. Kim, S.-H. Oh, C.-S. Kim \& C.-W. Park & 갈기기름나물 & Lee1805002 \\
\hline & Myrsinaceae 자금우과 & & \\
\hline 289 & Ardisia crenata Sims & 백량금 & KHB1435477 \\
\hline \multirow[t]{2}{*}{290} & Ardisia japonica (Thunb.) Blume & 자금우 & KHB1360258 \\
\hline & Primulaceae 앵초과 & & \\
\hline 291 & Anagallis arvensis var. caerulea (L.) Gouan & 뚜껑별꽃 & KHB1435795 \\
\hline 292 & Lysimachia barystachys Bunge & 까치수염 & KHB1363275 \\
\hline 293 & Lysimachia japonica Thunb. & 좀가지풀 & KHB1360234 \\
\hline \multirow[t]{2}{*}{294} & Lysimachia mauritiana Lam. & 갯까치수염 & KHB1360300 \\
\hline & Ebenaceae 감나무과 & & \\
\hline \multirow[t]{2}{*}{295} & Diospyros kaki Thunb. & 감나무 & Son0569 \\
\hline & Styracaceae 때죽나무과 & & \\
\hline \multirow[t]{2}{*}{296} & Styrax japonicus Siebold \& Zucc. & 때죽나무 & Son0793 \\
\hline & Symplocaceae 노린재나무과 & & \\
\hline 297 & Symplocos chinensis f. pilosa (Nakai) Ohwi & 노린재나무 & KHB1374388 \\
\hline \multirow[t]{2}{*}{298} & Symplocos tanakana Nakai & 검노린재나무 & KHB1435837 \\
\hline & Oleaceae 물푸레나무과 & & \\
\hline 299 & Ligustrum japonicum Thunb. & 광나무 & KHB1435786 \\
\hline \multirow[t]{2}{*}{300} & Ligustrum lucidum Aiton & 당광나무 & Son0519 \\
\hline & Apocynaceae 협죽도과 & & \\
\hline 301 & Nerium oleander L. & 협죽도 & KHB1369486 \\
\hline \multirow[t]{2}{*}{302} & Trachelospermum asiaticum (Siebold \& Zucc.) Nakai & 마삭줄 & KHB1436673 \\
\hline & Asclepiadaceae 박주가리과 & & \\
\hline 303 & Cynanchum japonicum Morr. \& Decne. & 덩굴민백미꽃 & KHB1435627 \\
\hline 304 & Cynanchum paniculatum (Bunge) Kitag. & 산해박 & KHB1436649 \\
\hline 305 & Cynanchum wilfordii (Maxim.) Hemsl. & 큰조롱 & KHB1436624 \\
\hline \multirow[t]{2}{*}{306} & Metaplexis japonica (Thunb.) Makino & 박주가리 & Hong8793 \\
\hline & Rubiaceae 꼭두서니과 & & \\
\hline 307 & Galium gracilens (A. Gray) Makino & 좀네잎갈퀴 & KHB1436635 \\
\hline 308 & Galium pogonanthum Franch. \& Sav. & 산갈퀴 & KHB1360257 \\
\hline 309 & Galium spurium var. echinospermon (Wallr.) Hayek & 갈퀴덩굴 & KHB1436685 \\
\hline 310 & Galium verum var. asiaticum Nakai & 솔나물 & KHB1363278 \\
\hline 311 & Hedyotis biflora var. parvifolia Hook. \& Arn. & 낚시돌풀 & KHB1435651 \\
\hline 312 & Paederia scandens (Lour.) Merr. & 계요등 & KHB1451093 \\
\hline 313 & Rubia akane Nakai & 꼭두서니 & KHB 1451088 \\
\hline \multirow[t]{2}{*}{314} & Rubia cordifolia var. pratensis Maxim. & 갈퀴꼭두서니 & KHB1435629 \\
\hline & Convolvulaceae 메꽃과 & & \\
\hline 315 & Calystegia dahuricus (Herb.) Choisy & 선메꽃 & KHB1436544 \\
\hline 316 & Calystegia sepium var. japonicum (Choisy) Makino & 메꽃 & КHB1440970 \\
\hline 317 & Calystegia soldanella (L.) Roem. \& Schultb. & 갯메꽃 & KHB1360298 \\
\hline
\end{tabular}


Appendix 1. Continued.

\begin{tabular}{|c|c|c|c|}
\hline 318 & Cuscuta japonica Choisy & 새삼 & KHB1435613 \\
\hline 319 & Dichondra repens Forster & 아욱메풀 & KHB1440908 \\
\hline 320 & Ipomoea hederacea var. integriuscula A. Gray & 둥근잎미국나팔꽃 & Hong1020703 \\
\hline 321 & Ipomoea purpurea Roth & 둥근잎나팔꽃 & Hon120711 \\
\hline \multirow[t]{2}{*}{322} & Quamoclit pennata (Desr.) Bojer & 유홍초 & KHB1435632 \\
\hline & Boraginaceae 지치과 & & \\
\hline 323 & Argusia sibirica (L.) Dandy & 모래지치 & KHB1360308 \\
\hline 324 & Bothriospermum tenellum (Hornem.) Fisch. \& C. A. Mey. & 꽃받이 & KHB1435868 \\
\hline 325 & Lithospermum zollingeri A.DC. & 반디지치 & KHB1435864 \\
\hline \multirow[t]{2}{*}{326} & Trigonotis peduncularis (Trevir.) Benth. ex Hemsl. & 꽃마리 & KHB1435958 \\
\hline & Verbenaceae 마편초과 & & \\
\hline 327 & Callicarpa japonica Thunb. & 작살나무 & Son0803 \\
\hline 328 & Callicarpa japonica var. luxurians Rehder & 왕작살나무 & KHB1440937 \\
\hline 329 & Caryopteris incana (Thunb.) Miq. & 층꽃나무 & KHB1435497 \\
\hline 330 & Clerodendrum trichotomum Thunb. & 누리장나무 & KHB1451046 \\
\hline \multirow[t]{2}{*}{331} & Vitex rotundifolia L.f. & 순비기나무 & $O B-P L-180300186$ \\
\hline & Labiatae 꿀풀과 & & \\
\hline 332 & Ajuga decumbens Thunb. & 금창초 & KHB1435969 \\
\hline 333 & Clinopodium chinense var. parviflorum (Kudo) Hara & 층층이꽃 & KHB1450927 \\
\hline 334 & Clinopodium chinense var. shibetchense (H. Lev.) Koidz. & 산층층이 & Lee1805017 \\
\hline 335 & Isodon inflexus (Thunb.) Kudo & 산박하 & KHB1451096 \\
\hline 336 & Isodon inflexus var. canescens (Nakai) Kudo & 털산박하 & KHB1373857 \\
\hline 337 & Lamium amplexicaule $\mathrm{L}$. & 광대나물 & KHB1435822 \\
\hline 338 & Leonurus japonicus Houtt. & 익모초 & KHB1441012 \\
\hline 339 & Prunella vulgaris var. lilacina Nakai & 꿀풀 & KHB1436686 \\
\hline 340 & Scutellaria indica $\mathrm{L}$. & 골무꽃 & Hong8871 \\
\hline \multirow[t]{2}{*}{341} & Scutellaria pekinensis var. transitra (Makino) Hara & 산골무꽃 & KHB1440959 \\
\hline & Solanaceae 가지과 & & \\
\hline 342 & Datura stramonium L. & 흰독말풀 & KHB1435390 \\
\hline 343 & Lycium chinense Mill. & 구기자나무 & Hong1020562 \\
\hline 344 & Solanum lyratum Thunb. & 배풍등 & KHB1369469 \\
\hline \multirow[t]{2}{*}{345} & Solanum nigrum $\mathrm{L}$. & 까마중 & KHB1435393 \\
\hline & Scrophulariaceae 현삼과 & & \\
\hline 346 & Rehmannia glutinosa (Gaertn.) Libosch. ex Steud. & 지황 & $O B-P L-180300192$ \\
\hline 347 & Siphonostegia chinensis Benth. & 절국대 & KHB1545060 \\
\hline 348 & Veronica arvensis $\mathrm{L}$. & 선개불알풀 & KHB1435857 \\
\hline 349 & Veronica hederaefolia $\mathrm{L}$. & 눈개불알풀 & KHB1435965 \\
\hline 350 & Veronica linariifolia Pall. ex Link & 꼬리풀 & KHB1435558 \\
\hline \multirow[t]{2}{*}{351} & Veronica persica Poir. & 큰개불알풀 & KHB1435846 \\
\hline & Acanthaceae 쥐꼬리망초과 & & \\
\hline 352 & Justicia procumbens $\mathrm{L}$. & 쥐꼬리망초 & KHB 1435378 \\
\hline
\end{tabular}


Appendix 1. Continued.

Orobanchaceae 열당과

353 Aeginetia indica $\mathrm{L}$.

야고

KHB1374271

Phrymaceae 파리풀과

354 Phryma leptostachya var. asiatica $\mathrm{H}$. Hara

파리풀

KHB1440967

Plantaginaceae 질경이과

355 Plantago asiatica L.

질경이

KHB1440820

356 Plantago camtschatica Cham. ex Link

개질경이

KHB1360304

357 Plantago lanceolata L.

창질경이

KHB1440896

358 Plantago major var. japonica (Franch. \& Sav.) Miyabe

왕질경이

Lee1805022

Caprifoliaceae 인동과

359 Lonicera japonica Thunb.

인동덩굴

KHB1360574

360 Lonicera japonica var. repens (Siebold) Rehder

털인동

KHB1436053

361 Sambucus sieboldiana (Miq.) Blume ex Graebn.

덧나무

KHB1362994

362 Viburnum odoratissimum var. awabuki (K.Koch) Zabel ex Rumpler

아왜나무

KHB1436054

Campanulaceae 초롱꽃과

363 Adenophora verticillata Fisch.

층층잔대

KHB1441006

364 Wahlenbergia marginata (Thunb.) A. DC.

애기도라지

KHB1363157

Compositae 국화과

365 Artemisia capillaris Thunb.

사철쑥

KHB1435366

366 Artemisia feddei H.Lev. \& Vaniot

뺑쑥

KHB 1435460

367 Artemisia princeps Pamp.

쑥

KHB 1435648

368 Artemisia rubripes Nakai

덤불쑥

Lee1805011

369 Aster arenarius (Kitam.) Nemoto

섬갯쑥부쟁이

Son 3713

370 Aster hispidus Thunb.

갯쑥부쟁이

KHB1436061

371 Aster pilosus Willd.

미국쑥부쟁이

KHB1451051

372 Aster sphathulifolius Maxim.

해국

KHB1435625

373 Aster subulatus Michx.

비짜루국화

KHB1451062

374 Aster yomena (Kitam.) Honda

쑥부쟁이

KHB 1374274

375 Bidens bipinnata $\mathrm{L}$.

376 Bidens biternata (Lour.) Merr. \& Sherff ex Sherff

도깨비바늘

KHB1435605

377 Bidens pilosa L.

털도깨비바늘

$O B-P L-180300216$

378 Bidens tripartita $\mathrm{L}$.

울산도깨비바늘

KHB1435397

379 Carpesium cernuum L.

가막사리

KHB1374409

380 Carpesium divaricatum Siebold \& Zucc.

좀담배풀

Lee1805026

381 Centipeda minima (L.) A. Br. \& Asch.

긴담배풀

KHB1435494

382 Cirsium japonicum var. maackii (Maxim.) Matsum.

중대가리풀

KHB1435430

383 Cirsium japonicum var. spinossimum Kitam.

엉겅퀴

KHB 1440867

384 Conyza canadensis (L.) Cronquist

가시엉겅퀴

Lee1805001

385 Conyza sumatrensis E.Walker

망초

KHB1435434

386 Cosmos bipinnatus Cav.

큰망초

KHB1435414

387 Crepidiastrum denticulatum (Houtt.) Pak \& Kawano

코스모스

KHB1435614

이고들빼기

KHB1435654 
Appendix 1. Continued.

\begin{tabular}{|c|c|c|c|}
\hline 388 & Crepidiastrum lanceolatum (Houtt.) Nakai & 갯고들빼기 & KHB1374385 \\
\hline 389 & Crepidiastrum sonchifolium (Bunge) Pak \& Kawano & 고들빼기 & KHB1436073 \\
\hline 390 & Dendranthema boreale (Makino) Ling ex Kitam. & 산국 & KHB1360576 \\
\hline 391 & Dendranthema indicum (L.) DesMoul. & 감국 & Son0722 \\
\hline 392 & Dendranthema zawadskii var. latilobum (Maxim.) Kitam. & 구절초 & KHB1360587 \\
\hline 393 & Dendranthema zawadskii var. yezoense (Maek.) Y. M. Lee \& H. J. Choi & 남구절초 & KHB1436681 \\
\hline 394 & Eclipta prostrata (L.) L. & 한련초 & KHB1374400 \\
\hline 395 & Erechtites hieracifolia Raf. & 붉은서나물 & KHB1435658 \\
\hline 396 & Erigeron annuus (L.) Pers. & 개망초 & KHB1436007 \\
\hline 397 & Eupatorium japonicum Thunb. & 등골나물 & KHB1374255 \\
\hline 398 & Eupatorium lindleyanum DC. & 골등골나물 & KHB1369462 \\
\hline 399 & Farfugium japonicum (L.) Kitam. & 털머위 & KHB1435835 \\
\hline 400 & Gnaphalium affine D. Don & 떡쑥 & KHB1440936 \\
\hline 401 & Gnaphalium calviceps Fernald & 선풀솜나물 & KHB1436538 \\
\hline 402 & Gnaphalium japonicum Thunb. & 풀솜나물 & KHB1440892 \\
\hline 403 & Gnaphalium purpureum L. & 자주풀솜나물 & KHB1440885 \\
\hline 404 & Hemistepta lyrata Bunge & 지칭개 & КHB1440893 \\
\hline 405 & Hypochaeris radicata $\mathrm{L}$. & 서양금혼초 & KHB1360581 \\
\hline 406 & Ixeridium dentatum (Thunb.) Tzvelev & 씀바귀 & KHB1435917 \\
\hline 407 & Ixeris debilis (Thunb.) A. Gray & 벋음씀바귀 & KHB1440810 \\
\hline 408 & Lactuca indica f. indivisa (Makino) Hara & 가는잎왕고들빼기 & KHB1435484 \\
\hline 409 & Lactuca indica $\mathrm{L}$. & 왕고들빼기 & KHB1435451 \\
\hline 410 & Leibnitzia anandria (L.) Turcz. & 솜나물 & KHB1435918 \\
\hline 411 & Petasites japonicus (Siebold \& Zucc.) Maxim. & 머위 & Lee1805014 \\
\hline 412 & Picris hieracioides var. koreana Kitam. & 쇠서나물 & Lee1805020 \\
\hline 413 & Senecio vulgaris $\mathrm{L}$. & 개쑥갓 & KHB1440927 \\
\hline 414 & Sigesbeckia glabrescens (Makino) Makino & 진득찰 & KHB1374412 \\
\hline 415 & Sigesbeckia orientalis L. & 제주진득찰 & KHB1435602 \\
\hline 416 & Solidago virgaurea subsp. asiatica Kitam. ex Hara & 미역취 & KHB1435493 \\
\hline 417 & Sonchus asper (L.) Hill & 큰방가지똥 & KHB1440965 \\
\hline 418 & Sonchus brachyotus DC. & 사데풀 & KHB1450947 \\
\hline 419 & Sonchus oleraceus L. & 방가지똥 & KHB1360571 \\
\hline 420 & Taraxacum coreanum Nakai & 흰민들레 & KHB1435854 \\
\hline 421 & Taraxacum mongolicum Hand.-Mazz. & 털민들레 & KHB1436051 \\
\hline 422 & Taraxacum officinale Weber & 서양민들레 & KHB1436052 \\
\hline 423 & Tephroseris kirilowii (Turcz. ex DC.) Holub & 솜방망이 & KHB1436072 \\
\hline \multirow[t]{2}{*}{424} & Youngia japonica (L.) DC. & 뽀리뱅이 & KHB1435980 \\
\hline & Zosteraceae 거머리말과 & & \\
\hline \multirow[t]{2}{*}{425} & Zostera marina $\mathrm{L}$. & 거머리말 & KHB1342300 \\
\hline & Liliaceae 백합과 & & \\
\hline 426 & Allium macrostemon Bunge & 산달래 & KHB1440935 \\
\hline
\end{tabular}


Appendix 1. Continued.

\begin{tabular}{|c|c|c|c|}
\hline 427 & Allium pseudojaponicum Makino & 갯부추 & KHB1436592 \\
\hline 428 & Allium sacculiferum Maxim. & 참산부추 & KHB1369479 \\
\hline 429 & Allium thunbergii G. Don & 산부추 & KHB1534172 \\
\hline 430 & Allium tuberosum Rottler ex Spreng. & 부추 & KHB1451022 \\
\hline 431 & Asparagus cochinchinensis (Lour.) Merr. & 천문동 & KHB1440853 \\
\hline 432 & Asparagus schoberioides Kunth & 비짜루 & KHB1360168 \\
\hline 433 & Disporum uniflorum Baker & 윤판나물 & $O B-P L-180300287$ \\
\hline 434 & Hemerocallis fulva (L.) L. & 원추리 & KHB1436631 \\
\hline 435 & Hemerocallis hakuunensis Nakai & 백 운산원추리 & Hong 8815 \\
\hline 436 & Hemerocallis thunbergii Baker & 노랑원추리 & Son0688 \\
\hline 437 & Lilium lancifolium Thunb. & 참나리 & KHB1373866 \\
\hline 438 & Lilium leichtlinii var. maximowiczii (Regel) Baker & 중나리 & $O B-P L-180300290$ \\
\hline 439 & Liriope platyphylla $\mathrm{F}$. T. Wang \& T. Tang & 맥문동 & KHB1435568 \\
\hline 440 & Ophiopogon jaburan (Kunth) Lodd. & 맥문아재비 & KHB1451040 \\
\hline 441 & Ophiopogon japonicus var. umbrosus Maxim. & 실맥문동 & Son0819 \\
\hline 442 & Scilla scilloides (Lind1.) Druce & 무릇 & KHB1435653 \\
\hline 443 & Smilax china L. & 청미래덩굴 & KHB1435843 \\
\hline 444 & Smilax riparia var. ussuriensis (Regel) Hara \& T. Koyama & 밀나물 & KHB1373883 \\
\hline \multirow[t]{2}{*}{445} & Smilax sieboldii f. intermis (Nakai) Hara & 민청가시덩굴 & Son0551 \\
\hline & Amaryllidaceae 수선화과 & & \\
\hline \multirow[t]{2}{*}{446} & Zephyranthes candida (Lindl.) Herb. & 흰꽃나도사프란(식재) & KHB1435650 \\
\hline & Dioscoreaceae 마과 & & \\
\hline 447 & Dioscorea japonica Thunb. & 참마 & Son0797 \\
\hline 448 & Dioscorea nipponica Makino & 부채마 & KHB1435386 \\
\hline 449 & Dioscorea polystachya Turcz. & 마 & KHB1436628 \\
\hline \multirow[t]{2}{*}{450} & Dioscorea quinqueloba Thunb. & 단풍마 & KHB1369474 \\
\hline & Iridaceae 붓꽃과 & & \\
\hline \multirow[t]{2}{*}{451} & Sisyrinchium angustifolium Mill. & 등심붓꽃 & KHB1436656 \\
\hline & Juncaceae 골풀과 & & \\
\hline 452 & Juncus bufonius L. & 애기골풀 & Son3839 \\
\hline 453 & Juncus diastrophanthus Buchenau & 별날개골풀 & KHB1440851 \\
\hline 454 & Juncus effusus var. decipiens Buchenau & 골풀 & KHB1440858 \\
\hline 455 & Juncus gracillimus (Buchenau) V. I. Krecz. \& Gontsch. & 물골풀 & KHB1440871 \\
\hline 456 & Juncus haenkei E.Mey. & 갯골풀 & Son3789 \\
\hline 457 & Juncus setchuensis var. effusoides Buchenau & 푸른갯골풀 & KHB1435416 \\
\hline \multirow[t]{2}{*}{458} & Luzula capitata (Miq.) Miq. & 꿩의밥 & KHB1436045 \\
\hline & Commelinaceae 닭의장풀과 & & \\
\hline 459 & Aneilema keisak Hassk. & 사마귀풀 & KHB1435419 \\
\hline 460 & Commelina communis L. & 닭의장풀 & KHB1436660 \\
\hline \multirow[t]{2}{*}{461} & Commelina communis var. hortensis Makino & 큰닭의장풀 & Lee1805028 \\
\hline & Gramineae 벼과 & & \\
\hline
\end{tabular}


Appendix 1. Continued.

\begin{tabular}{|c|c|c|c|}
\hline 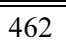 & Agropyron ciliare (Trin.) Franch. & 속털개밀 & KHB1436024 \\
\hline 463 & Agropyron tsukushiense var. transiens (Hack.) Ohwi & 개밀 & KHB1436028 \\
\hline 464 & Alopecurus aequalis Sobol. & 뚝새풀 & KHB1360314 \\
\hline 465 & Arthraxon hispidus (Thunb.) Makino & 조개풀 & $O B-P L-180300247$ \\
\hline 466 & Arundinella hirta (Thunb.) Koidz. & 새 & KHB1450961 \\
\hline 467 & Arundinella hirta var. ciliata Koidz. & 털새 & KHB1450953 \\
\hline 468 & Avena fatua $\mathrm{L}$. & 메귀리 & КHB1360306 \\
\hline 469 & Brachypodium sylvaticum (Huds.) P. Beauv. & 숲개밀 & КHB1070521 \\
\hline 470 & Briza minor L. & 방울새풀 & KHB1440811 \\
\hline 471 & Bromus unioloides H. B. \& K. & 큰이삭풀 & KHB1436063 \\
\hline 472 & Calamagrostis arundinacea (L.) Roth & 실새풀 & КHB1451071 \\
\hline 473 & Calamagrostis epigeios (L.) Roth & 산조풀 & Son3782 \\
\hline 474 & Capillipedium parviflorum (R.Br.) Stapf & 나도기름새 & KHB1435498 \\
\hline 475 & Cleistogenes hackelii (Honda) Honda & 대새풀 & KHB1451063 \\
\hline 476 & Cymbopogon tortilis var. goeringii (Steud.) Hand.-Mazz. & 개솔새 & KHB1435362 \\
\hline 477 & Cynodon dactylon (L.) Pers. & 우산잔디 & KHB1435402 \\
\hline 478 & Dactylis glomerata $\mathrm{L}$. & 오리새 & KHB1435859 \\
\hline 479 & Digitaria ciliaris (Retz.) Koel. & 바랭이 & КHB1435617 \\
\hline 480 & Digitaria violascens Link & 민바랭이 & KHB1435461 \\
\hline 481 & Echinochloa crusgalli (L.) P. Beauv. & 돌피 & KHB1435541 \\
\hline 482 & Echinochloa crusgalli var. praticola Ohwi & 좀돌피 & КHB1450946 \\
\hline 483 & Echinochloa utilis Ohwi \& Yabuno & 피 & P133247 \\
\hline 484 & Eleusine indica (L.) Gaertn. & 왕바랭이 & KHB1435563 \\
\hline 485 & Eriochloa villosa (Thunb.) Kunth & 나도개피 & KHB1435355 \\
\hline 486 & Festuca arundinacea Schreb. & 큰김의털 & KHB1435924 \\
\hline 487 & Festuca myuros L. & 들묵새 & КHB1436610 \\
\hline 488 & Festuca ovina $\mathrm{L}$. & 김의털 & KHB1435975 \\
\hline 489 & Festuca parvigluma Steud. & 김의털아재비 & КHB1435946 \\
\hline 490 & Imperata cylindrica var. koenigii (Retz.) Pilg. & 띠 & KHB1436679 \\
\hline 491 & Ischaemum crassipes (Steud.) Thell. & 쇠보리 & KHB1435655 \\
\hline 492 & Koeleria cristata (L.) Pers. & 도랭이피 & KHB1360170 \\
\hline 493 & Lolium multiflorum Lam. & 쥐보리 & KHB1360305 \\
\hline 494 & Lolium perenne L. & 호밀풀 & Son3674 \\
\hline 495 & Microstegium vimineum (Trin.) A.Camus & 나도바랭이새 & $O B-P L-180300261$ \\
\hline 496 & Microstegium vimineum var. imberbe (Nees ex Steud.) Honda & 큰듬성이삭새 & KHB1451073 \\
\hline 497 & Miscanthus sinensis Andersson & 참억새 & KHB1435422 \\
\hline 498 & Miscanthus sinensis f. gracillimus (Hitchc.) Ohwi & 가는잎억새 & KHB1450962 \\
\hline 499 & Miscanthus sinensis var. purpurascens (Andersson) Rendle & 억새 & KHB1450923 \\
\hline 500 & Oplismenus undulatifolius (Ard.) P. Beauv. & 주름조개풀 & KHB1435579 \\
\hline 501 & Oplismenus undulatifolius var. japonicus (Steud.) Koidz. & 민주름조개풀 & KHB1435389 \\
\hline 502 & Parapholis incurva (L.) C. E. Hubb. & 뿔이삭풀 & KHB1435813 \\
\hline
\end{tabular}


Appendix 1. Continued.

\begin{tabular}{|c|c|c|c|}
\hline 503 & Paspalum dilatatum Poir. & 큰참새피 & KHB1435637 \\
\hline 504 & Paspalum thunbergii Kunth ex Steud. & 참새피 & KHB1440882 \\
\hline 505 & Pennisetum alopecuroides (L.) Spreng. & 수크령 & KHB1435623 \\
\hline 506 & Phacelurus latifolius (Steud.) Ohwi & 모새달 & $O B-P L-180300266$ \\
\hline 507 & Phacelurus latifolius f. angustifolius (Debeaux) Kitag. & 가는잎모새달 & KHB1440846 \\
\hline 508 & Phragmites communis Trin. & 갈대 & KHB1435638 \\
\hline 509 & Poa acroleuca var. submoniliformis Makino & 마디포아풀 & KHB1435937 \\
\hline 510 & Poа аппиа L. & 새포아풀 & KHB1435957 \\
\hline 511 & Poa sphondylodes Trin. & 포아풀 & KHB1435885 \\
\hline 512 & Polypogon fugax Nees ex Steud. & 쇠돌피 & KHB1436655 \\
\hline 513 & Polypogon monspeliensis (L.) Desf. & 갯쇠돌피 & KHB1436699 \\
\hline 514 & Puccinellia nipponica Ohwi & 갯꾸러미풀 & KHB 1440848 \\
\hline 515 & Sacciolepis indica (L.) Chase & 좀물뚝새 & KHB1435524 \\
\hline 516 & Sacciolepis indica var. oryzetorum (Makino) Ohwi & 물뚝새 & KHB1435591 \\
\hline 517 & Sasa coreana Nakai & 신이대 & Son0756 \\
\hline 518 & Setaria faberii Herrm. & 가을강아지풀 & KHB1440918 \\
\hline 519 & Setaria glauca (L.) P.Beauv. & 금강아지풀 & KHB1451066 \\
\hline 520 & Setaria viridis (L.) P.Beauv. & 강아지풀 & KHB1451075 \\
\hline 521 & Setaria viridis var. pachystachys (Franch. \& Sav.) Makino \& Nemoto & 갯강아지풀 & KHB1435358 \\
\hline 522 & Spodipogon cotulifer (Thunb.) Hack. & 기름새 & KHB1435473 \\
\hline 523 & Sporobolus fertilis (Steud.) Clayton & 쥐꼬리새풀 & KHB1451053 \\
\hline 524 & Themeda triandra var. japonica (Willd.) Makino & 솔새 & KHB1435574 \\
\hline 525 & Trisetum bifidum (Thunb.) Ohwi & 잠자리피 & KHB1440805 \\
\hline 526 & Zoysia japonica Steud. & 잔디 & KHB1436004 \\
\hline \multirow[t]{2}{*}{527} & Zoysia sinica Hance & 갯잔디 & KHB1440952 \\
\hline & Araceae 천남성과 & & \\
\hline 528 & Arisaema heterophyllum Blume & 두루미천남성 & Son0713 \\
\hline 529 & Arisaema ringens (Thunb.) Schott & 큰천남성 & KHB1436079 \\
\hline 530 & Arisaema thunbergii Blume & 무늬천남성 & KHB1404849 \\
\hline 531 & Pinellia ternata (Thunb.) Breitenb. & 반하 & KHB1373884 \\
\hline \multirow[t]{2}{*}{532} & Pinellia tripartita (Blume) Schott & 대반하 & KHB1369475 \\
\hline & Cyperaceae 사초과 & & \\
\hline 533 & Bulbostylis densa (Wall.) Hand.-Mazz. & 꽃하늘지기 & $P 133374$ \\
\hline 534 & Carex boottiana Hook. \& Arn. & 밀사초 & KHB1436010 \\
\hline 535 & Carex breviculmis R. Br. & 청사초 & KHB1360235 \\
\hline 536 & Carex breviculmis var. fibrillosa Kuk. & 갯청사초 & Son3757 \\
\hline 537 & Carex gibba Wahlenb. & 나도별사초 & KHB1436539 \\
\hline 538 & Carex lanceolata Boott & 그늘사초 & KHB1435797 \\
\hline 539 & Carex lenta D. Don & 줄사초 & KHB1360583 \\
\hline 540 & Carex mitrata Franch. var. aristata Ohwi & 까락겨사초 & KHB1435968 \\
\hline 541 & Carex polyschoena H. Lev. \& Vaniot & 가지청사초 & KHB1435970 \\
\hline
\end{tabular}


Appendix 1. Continued.

\begin{tabular}{|c|c|c|c|}
\hline 542 & Cladium chinense Nees & 층층고랭이 & KHB1435584 \\
\hline 543 & Cyperus amuricus Maxim. & 방동사니 & KHB1435660 \\
\hline 544 & Cyperus cyperoides (L.) Kuntze & 방동사니아재비 & KHB1440877 \\
\hline 545 & Cyperus difformis $\mathrm{L}$. & 알방동사니 & KHB1435585 \\
\hline 546 & Cyperus iria $\mathrm{L}$. & 참방동사니 & KHB1450940 \\
\hline 547 & Cyperus microiria Steud. & 금방동사니 & KHB1450950 \\
\hline 548 & Cyperus polystachyos Rottb. & 갯방동사니 & KHB1435662 \\
\hline 549 & Cyperus rotundus $\mathrm{L}$. & 향부자 & KHB1435372 \\
\hline 550 & Cyperus sanguinolentus Vahl & 방동사니대가리 & KHB1435570 \\
\hline 551 & Eleocharis mamillata var. cyclocarpa Kitag. & 물꼬챙이골 & KHB1360310 \\
\hline 552 & Fimbristylis ferruginea var. sieboldii (Miq.) Ohwi & 갯하늘지기 & KHB1435608 \\
\hline 553 & Fimbristylis tristachya var. subbispicata (Nees \& Meyen) T. Koyama & 꼴하늘지기 & KHB1435527 \\
\hline 554 & Kyllinga brevifolia Rottb. & 파대가리 & KHB1360569 \\
\hline \multirow[t]{2}{*}{555} & Scirpus planiculmis F.Schmidt & 좀매자기 & KHB1440809 \\
\hline & Orchidaceae 난초과 & & \\
\hline 556 & Epipactis thunbergii A.Gray & 닭의난초 & KHB1436607 \\
\hline 557 & Platanthera mandarinorum var. brachycentron (Franch. \& Sav.) Koidz. ex Ohwi & 산제비란 & KHB1436636 \\
\hline 558 & Spiranthes sinensis (Pers.) Ames & 타래난초 & KHB1436633 \\
\hline
\end{tabular}

